(c) American Dairy Science Association, 2006.

\title{
The American Dairy Science Association: The Fourth Twenty-Five Years, 1981-2005 ${ }^{1}$
}

\author{
D. C. Beitz ${ }^{\star 2}$ and L. J. Boyd $\dagger^{3}$ \\ *Nutritional Physiology Group, Department of Animal Science, lowa State University, Ames 50011-3150 \\ †Department of Animal and Dairy Science, University of Georgia, Athens 30602
}

\begin{abstract}
Great leaders have stepped forward to successfully lead the American Dairy Science Association (ADSA) through its fourth 25-yr period of its 100-yr history. Rather than meeting on college campuses, the annual meetings of the ADSA are now held at convention centers that are easily accessible, and are usually held jointly with the American Society of Animal Science. Besides the scientific and social exchanges, regular members and students are recognized for their professional excellence with awards at these meetings. The Student Affiliate Division continues to hold an active meeting along with the parent society. The last $25 \mathrm{yr}$ experienced the termination of the Purina Mills Fellowship Program after 52 yr of support for graduate students. The establishment of an ADSA Foundation has increased resources for enhancement of the ADSA. Newly established DISCOVER Conferences are recipients of Foundation support. The last 25-yr period experienced the development of a formal business arrangement with the American Society of Animal Science and Poultry Science Association that resulted in the establishment of the Federation of Animal Science Societies (FASS), which now houses the 3 societies in its building in Savoy, IL. The Journal of Dairy Science has become the leading journal for publication of dairy science research in the world. The Journal as well as the membership of the ADSA has become increasingly internationalized. Truly, the ADSA has much to celebrate because of its successes in mission during the fourth $25 \mathrm{yr}$ of its history. Its membership can be confident as the ADSA begins its second century of service to the dairy industry.
\end{abstract}

Key words: American Dairy Science Association history, American Dairy Science Association Foundation,

Received June 30, 2005.

Accepted July 5, 2005.

${ }^{1}$ Publication of the Iowa Agricultural and Home Economics Experiment Station, Ames; Project Number 3801.

${ }^{2}$ Corresponding author: dcbeitz@iastate.edu

${ }^{3}$ Professor Emeritus.
Journal of Dairy Science, American Dairy Science Association annual meeting

\section{INTRODUCTION}

The American Dairy Science Association (ADSA) was founded in 1906 as the National Association of Dairy Instructors and Investigators in Urbana, IL. Thus, in 2006, the ADSA will have begun its second century of existence. The history of the first $50 \mathrm{yr}$ of the ADSA was described in much detail by G. Malcolm Trout in the Journal of Dairy Science in 1956. The next $25 \mathrm{yr}$ also were recorded in the same journal in 1981. What a valuable contribution G. M. Trout has made to our ADSA through his writings as historian of the Association! Trout received a special plaque during the Diamond Jubilee year of ADSA as a "token of our thanks and appreciation for his serving as a model member of ADSA for nearly 60 years and for his comprehensive historical accounts over the years." The business affairs of ADSA from its establishment in 1906 to 1985 were recorded by Walter L. Dunkley in the Journal of Dairy Science in 1989, and G. M. Trout published a review of ADSA Awards in 1961 in the same journal.

The first 75 yr of the ADSA saw growth in membership from the 19 charter members in July 1906 to nearly 2500 members, 100 student affiliates, and 2200 institutional members. With increased membership came a large budget to support the publication of the Journal of Dairy Science and a Board of Directors and staff to coordinate the activities and the arranging for the annual meeting scientific exchange and fellowship. The fourth 25-yr history of the century of existence of ADSA is summarized in this article. Special emphasis is placed on the people who made ADSA what it is today and the major changes in activities of the Association.

\section{ANNUAL MEETINGS}

The annual meetings of the ADSA were held on college campuses beginning in 1927 and continuing through 1997, with the exception of 1989 , for a total of 70 meetings (Table 1). The meeting sites provided the opportunity for attendees to become familiar with a 
Table 1. Location of and attendance at annual meetings of the American Dairy Science Association (ADSA), 1981-2005

\begin{tabular}{|c|c|c|c|c|c|c|c|c|}
\hline Meeting & Location & Year & Members & Nonmembers & Students & Spouses & Children & Total \\
\hline 77th & State College, PA & 1982 & 731 & 205 & 443 & 364 & 234 & 1,977 \\
\hline 78th & Madison, WI & 1983 & 855 & 296 & 437 & 344 & 179 & 2,101 \\
\hline 80th & Urbana, IL & 1985 & 801 & 246 & 455 & 227 & 126 & 1,855 \\
\hline 81 st & Davis, CA & 1986 & 764 & 266 & 294 & 247 & 110 & 1,681 \\
\hline 82nd & Columbia, MO & 1987 & 801 & 373 & 444 & 206 & 113 & 1,837 \\
\hline 86th & Logan, UT & 1991 & 766 & 161 & 349 & 195 & 94 & 1,567 \\
\hline 87th & Columbus, OH & 1992 & 851 & 175 & 390 & 205 & 122 & 1,743 \\
\hline 88th & College Park, MD & 1993 & 1,015 & 180 & 408 & 167 & 94 & 1,804 \\
\hline 89th & St. Paul, MN & 1994 & 1,900 & 412 & 828 & 317 & 105 & 3,562 \\
\hline 90th & Ithaca, NY & 1995 & 989 & 172 & 476 & 166 & 69 & 1,872 \\
\hline 91st & Corvallis, OR & 1996 & 1,139 & 361 & 297 & 187 & 79 & 2,063 \\
\hline 97th & Quebec City, Canada ${ }^{3,5}$ & 2002 & 1,667 & 336 & 832 & - & - & 2,835 \\
\hline 98th & Phoenix, $\mathrm{AZ}^{1,5}$ & 2003 & 1,549 & 229 & 789 & - & - & 2,567 \\
\hline 99th & St. Louis, $\mathrm{MO}^{4,5}$ & 2004 & 2,044 & 307 & 1,028 & - & - & 3,379 \\
\hline 100th & Cincinnati, $\mathrm{OH}^{1,5}$ & 2005 & 1,314 & 290 & 759 & - & - & 2,787 \\
\hline
\end{tabular}

${ }^{1}$ Joint meeting of ADSA and American Society of Animal Science (ASAS).

${ }^{2}$ Joint meeting of ADSA, ASAS, Poultry Science Association (PSA), and American Meat Science Association (AMSA).

${ }^{3}$ Joint meeting of ADSA, ASAS, and Canadian Society for Animal Science (CSAS).

${ }^{4}$ Joint meeting of ADSA, ASAS, and PSA.

${ }^{5}$ Meeting at a hotel complex and/or convention center. From 1998 to 2004, members include fellows, retirees, corporate members, speakers, and exhibitors; students include undergraduates, graduates, and postdoctorates. After 1999, spouses and children did not have to register for the annual meeting. Totals may include nonpaying registrants including speakers, fellows, exhibitors, and staff; therefore, totals may exceed the sum of the columns.

variety of college campuses and regions of the country. Many family vacations were planned in association with participation in the ADSA meetings. In 1978, the annual meeting (the 73rd) was held jointly with the American Society of Animal Science (ASAS). Because of its success, the impetus for holding future joint meetings became strong. A second joint ADSA/ASAS meeting (the 84th) was hosted by the University of Kentucky. This meeting was the first of many subsequent meetings held at a hotel or convention center complex. For several years, the Board of Directors of ADSA debated the advantages and disadvantages of holding the annual meetings at hotels and convention centers rather than at college campuses. The wish of members for more comfortable housing and meeting rooms, the many hours required to host an ADSA meeting on a college campus, and the large size of the meetings, especially the ones held jointly with ASAS, are 3 important reasons for the switch to convention centers despite the increased costs for attendees. The Board of Directors decided in 1995 that all future meetings would be held at convention sites that are easily accessible to major transportation systems. All meetings since the 93rd held in Denver in 1998 have been at convention centers, and most have been held jointly with the ASAS. Commercial exhibits were added to enhance the annual meetings in 1996. Submission of abstracts electronically was initiated for the 1998 meeting.

Attendance by members has ranged from about 550 to about 950 with an average over the 25-yr period of over 800 for ADSA-only meetings (Table 1). Nonmembers, student affiliates, spouses, and children raise the average attendance to over 1700 per ADSA-only meeting. Recording the attendance by ADSA members at joint meetings has been problematic. Because of the movement from college campuses and a decrease in organized spouse and children activities, attendance by spouses and children has decreased in recent years.

Throughout this 25-yr period, the Student Affiliate Division (SAD) held its annual meeting and awards luncheon in association with the annual meeting of its parent society. The SAD organized its own scientific and social program, paper presentation contest, display of local club activities, and tours of local dairy industries. The SAD is described more fully later in this article. The enthusiasm of these young members pro- 
vides the parent association much optimism for its future.

\section{THE ADSA BELL}

The story of acquiring the Swiss bell that is now the "ADSA Bell" was described by S. D. Musgrave in the Journal of Dairy Science. Briefly, Musgrave offered G. $\mathrm{H}$. Wise (the 49th president of ADSA) the Swiss Bell during a casual meeting on Peachtree Street in Atlanta, GA, with the statement "I dare you to open the annual meeting by ringing this bell and, if you will, I will give it to you to use and to pass on to succeeding presidents to use-if they wish to do so." The Bell had originally been given to Musgrave by $\mathrm{H}$. W. Cave, the 25 th president of ADSA. In fact, the Bell was used for the first time by G. H. Wise to open the 60th annual meeting of ADSA in Lexington, KY. Succeeding presidents have established a tradition now by opening all subsequent meetings of ADSA with the ringing of the Bell.

\section{GRADUATE STUDENT PAPER PRESENTATION CONTEST}

The Student Affiliate Committee (W. L. Slatter, chair, L. K. Crowe, L. R. Dowd, P. M. Reaves, and W. W. Snyder) proposed to the Board of Directors of ADSA in 1956 that "a technical paper writing and presentation competition for graduate students" be initiated. The Board appointed G. Salisbury, W. V. Price, and I. A. Gould to further evaluate the project and report to the Board in 1957 and again in 1958. In 1958, the Board instructed the Student Affiliate Committee to offer a contest for the Production Division on a trial basis in 1959 at the 54th annual meeting in Urbana, IL, and provided $\$ 100$ for the winner. Committee chair W. W. Snyder presented the top award at this initial contest to J. B. Stone and honorable mention awards to K. E. Ebner and E. W. Wickersham. Because of its success the first year, the contest was extended to the Dairy Foods Division the next year. The contest has been a popular event ever since its initiation. The complete list of first-place winners for each Division is presented in Table 2. A long-time sponsor for the contest has been the National Milk Producers Federation. In 2004, ADSA sponsored the Dairy Foods award. In 2005, Dairy Management Inc. sponsored the Dairy Foods award. The National Milk Producer's Federation continued to sponsor the Production award in 2004 and 2005. Besides the national Contest, the Southern and Northeast Branches sponsor Graduate Student Paper Competitions as well. For the national contest, a certificate and cash award is presented to first-, second-, and thirdplace winners in each Division, and all participants
Table 2. First-place winners at graduate student paper presentation contests $^{1}$

\begin{tabular}{|c|c|c|}
\hline Year & Dairy Foods Division & Production Division \\
\hline $1959^{2}$ & Not established & J. B. Stone \\
\hline 1960 & J. A. Jaynes & J. T. Huber \\
\hline 1961 & J. A. Schlegel & J. S. Sutton \\
\hline 1962 & R. L. Bradley Jr. & D. E. Otterby \\
\hline 1963 & M. E. Stiles & A. K. Fowler \\
\hline 1964 & P. Crater & D. P. Wesen \\
\hline 1965 & R. M. Parry Jr. & S. D. Aust \\
\hline 1966 & R. V. Josephson & D. R. Davis \\
\hline 1967 & B. Talbot & R. L. Lavker \\
\hline 1968 & P. B. McNulty & P. L. Senger \\
\hline 1969 & G. A. Reineccius & A. A. Simpson \\
\hline 1970 & R. L. Richter & M. F. Hutjens \\
\hline 1971 & P. E. Swenson & R. C. Bennett \\
\hline 1972 & P. E. Plantz & R. S. Patton \\
\hline 1973 & J. R. Uckert & G. L. Beardsley \\
\hline 1974 & J. B. White & R. J. Dennis \\
\hline 1975 & L. L. Bell & H. W. Polzin \\
\hline 1976 & T. M. Gilmore & S. C. Nickerson \\
\hline 1977 & D. E. Satternexx & J. A. Foley \\
\hline 1978 & J. M. Wingfield & L. B. Hansen \\
\hline 1979 & L. M. H. Reichenbach & W. McKenzie \\
\hline 1980 & C. Papa & L. E. Armentano \\
\hline 1981 & M. E. Sanders & G. E. Huston \\
\hline 1982 & F. C. Church & R. R. Lyle \\
\hline 1983 & S. M. Schellhaass & J. J. Veenhuizen \\
\hline 1984 & R. J. Verdi & D. J. Miller \\
\hline 1985 & A. R. Hill & L. M. Sordillo \\
\hline 1986 & J. Feirtag & M. J. VandeHaar \\
\hline 1987 & J. U. McGregor & W. M. Seymour \\
\hline 1988 & D. A. Romero & L. Jones \\
\hline 1989 & R. D. Byrne Jr. & C. Koehler \\
\hline 1990 & J. R. Broadbent & A. L. Van Eenennaa \\
\hline 1991 & G. A. Stegeman & J. M. Chow \\
\hline 1992 & A. Mustapha & C. R. Simmons \\
\hline 1993 & S. M. Tosh & P. W. Joyce \\
\hline 1994 & G. Allison & C. G. Aldrich \\
\hline 1995 & S. A. Walker & K. Shafer-Weaver \\
\hline 1996 & J. Kiely & R. C. Hovey \\
\hline 1997 & D. Low & K. Knowlton \\
\hline 1998 & L. E. Metzger & T. R. Mackle \\
\hline 1999 & Y. Ma & J. Wells \\
\hline 2000 & E. Dudley & F. Moreira \\
\hline 2001 & A. Hassan & E. Mosley \\
\hline 2002 & D. Bergmaier & L. Silva \\
\hline 2003 & D. A. Patel & S. Musters \\
\hline 2004 & S. A. O'Mahony ${ }^{3}$ & J. Kay \\
\hline 2005 & L. Davis-Rincker ${ }^{3}$ & C. Udayarajan \\
\hline
\end{tabular}

${ }^{1}$ Sponsored by The National Milk Producer's Federation.

${ }^{2}$ First-place winners before 1981 are presented here because of not being presented in previous historical publications.

${ }^{3}$ In 2004, ADSA sponsored the Dairy Foods award. In 2005, Dairy Management Inc. sponsored the Dairy Foods award. The National Milk Producer's Federation continued to sponsor the Production award in 2004 and 2005.

must be members of the ADSA and present their original research. This long-standing event promises to generate much interest and professional growth in future ADSA participants.

\section{ADSA AWARDS}

The ADSA continued to reward its members for their success in research, teaching, extension, and service 
activities. The Awards have been supported generously by a variety of industries and organizations as shown in Table 3. The previous 25-yr period began with 12 different awards in addition to the Graduate Student Paper Contest and the Purina Mills Fellowship program. Sponsors for some Awards changed, but the criteria for receiving the Awards have remained the same. During the same period, the Dairy Research Foundation Award and the Purina Mills Fellowship program were discontinued, the American Cyanamid Award began in 1986 and terminated in 1993, and the National Dairy Herd Improvement Association Award began in 1983 and terminated in 1989. The winners of the Awards, except the Fellow Award, the Graduate Student Paper Contest, the Purina Mills Fellowship, ADSA Foundation Scholar, and Genevieve Christen Undergraduate Student awards, are presented in Table 3. Winners of the latter awards are depicted elsewhere in this article. The members of the ADSA are indeed grateful to all the sponsors of all Awards so that excellence among our ADSA membership can be recognized in an appropriate and timely manner.

\section{FELLOWS PROGRAM}

After being thoroughly researched by the Internal Affairs Committee, ADSA initiated a Fellows program in 1997. Previous recipients of the Distinguished Service Award and the ADSA Award of Honor who were still professionally active and met the established criteria were named as Fellows to jump-start the Fellows program in 1997. The Fellows Award would recognize ADSA members who have rendered distinguished service to the dairy industry, to a dairy-related discipline, or to ADSA. The Fellow also must have had continuous professional membership in ADSA for at least 20 years and must be in good standing in the Association. The number selected each year will be limited to $0.2 \%$ of the membership. The names of Fellows inducted in 1997 and elected as Fellows since initiation of the program are listed in Table 4. Election to Fellow is one of the highest distinctions an ADSA member can achieve.

\section{LIFE MEMBERS}

Life Member status is granted to a professional member who meets 1 of these 2 criteria: 1) Professional member in good standing who retires after having been a member for 25 years or more, including time as a Student Affiliate Member, or 2) Professional member in good standing who has paid dues to the ADSA for $40 \mathrm{yr}$ or more, including time as a Student Affiliate Member. Life Members are not required to pay dues but are eligible to vote and have electronic access to

Table 3. American Dairy Science Association awards, 1981-2005

\begin{tabular}{llll}
\hline \multicolumn{4}{c}{$\begin{array}{c}\text { Alltech, Inc. Graduate Student Paper } \\
\text { Publication Award (1998) }\end{array}$} \\
1998 & B. E. Kegley & 2002 & A. Abu-Ghazaleh \\
1999 & M. A. Rudan & 2003 & F. Bargo \\
2000 & A. Hippen & 2004 & P. Kononoff \\
2001 & D. C. Donovan & 2005 & D. Z. Caraviello \\
& American Feed Industry Association (1948) \\
1981 & W. V. Chalupa & 1994 & W. W. Hoover \\
1982 & D. E. Bauman & 1995 & R. R. Grummer \\
1983 & R. L. Horst & 1996 & R. A. Erdman \\
1984 & H. F. Tyrrell & 1997 & G. A. Broderick \\
1985 & D. C. Beitz & 1998 & J. P. Goff \\
1986 & C. J. Sniffen & 1999 & T. Jenkins \\
1987 & J. W. Young & 2000 & G. A. Varga \\
1988 & C. E. Polan & 2001 & W. P. Weiss \\
1989 & D. J. Schingoethe & 2002 & J. K. Drackley \\
1990 & C. E. Coppock & 2003 & D. R. Mertens \\
1991 & M. D. Stern & 2004 & L. Armentano \\
1992 & D. L. Palmquist & 2005 & C. Schwab \\
1993 & J. B. Russell & &
\end{tabular}

Cargill Animal Nutrition Young Scientist Award (2004) (Formerly Agway, Inc. from 1983-2003)

\begin{tabular}{|c|c|c|c|}
\hline & & & \\
\hline 1983 & P. L. Senger & 1995 & L. M. Sordillo \\
\hline 1984 & R. D. Shanks & 1996 & I. Politis \\
\hline 1985 & R. L. Ax & 1997 & J. K. Drackley \\
\hline 1986 & R. M. Akers & 1998 & G. W. Rogers \\
\hline 1987 & R. L. Horst & 1999 & G. E. Dahl \\
\hline 1988 & S. C. Nickerson & 2000 & K. Plaut \\
\hline 1989 & S. P. Oliver & 2001 & M. A. McGuire \\
\hline 1990 & J. S. Stevenson & 2002 & R. A. Kohn \\
\hline 1991 & P. J. Hansen & 2003 & K. A. Weigel \\
\hline 1992 & J. P. McNamara & 2004 & C. van Tassell \\
\hline 1993 & B. W. McBride & 2005 & J. E. P. Santos \\
\hline 1994 & C. K. Reynolds & & \\
\hline & $\begin{array}{l}\text { DeLaval Dai } \\
\text { (Formerl. }\end{array}$ & sic & $\begin{array}{l}\text { vard (1951) } \\
\text { ri, Inc.) }\end{array}$ \\
\hline 1981 & B. Harris Jr. & 1994 & R. L. Bradley Jr. \\
\hline 1982 & D. L. Bath & 1995 & S. B. Spencer \\
\hline 1983 & R. D. Appleman & 1996 & F. W. Bodyfelt \\
\hline 1984 & R. W. Everett & 1997 & L. D. Guthrie \\
\hline 1985 & M. F. Hutjens & 1998 & R. Cady and M. Varner \\
\hline 1986 & J. C. Bruhn & 1999 & W. L. Crist \\
\hline 1987 & D. V. Armstrong & 2000 & L. E. Chase \\
\hline 1988 & A. N. Bringe & 2001 & M. L. Eastridge \\
\hline 1989 & W. T. Howard & 2002 & J. F. Smith \\
\hline 1990 & D. K. Bandler & 2003 & P. Hoffman \\
\hline 1991 & R. S. Adams & 2004 & R. Nebel \\
\hline 1992 & J. V. Chambers & 2005 & R. Shaver \\
\hline
\end{tabular}

1993

E. P. Call

DSM Food Specialties Award (2000)

(Formerly Pfizer Inc. Award 1959-1994;

Cultor Food Science Award in 1996; Gist-Brocades Award 1997-1999)

1981

1982

1983

1984

1985

1986

1987

1988

1989

1990

1991

1992

$\begin{array}{lll}\text { H. A. Morris } & 1993 & \text { P. S. Kindstedt } \\ \text { M. Kalab } & 1994 & \text { R. J. Baer } \\ \text { D. M. Irvine } & 1996 & \text { G. A. Somkuti } \\ \text { T. R. Klaenhammer } & 1997 & \text { J. F. Frank } \\ \text { R. J. Brown } & 1998 & \text { E. A. Foegeding } \\ \text { V. R. Harwalkar } & 1999 & \text { D. J. McMahon } \\ \text { C. H. Amundson } & 2000 & \text { J. Steele } \\ \text { C. L. Hicks } & 2001 & \text { C. LaCroix } \\ \text { T. Richardson } & 2002 & \text { J. R. Broadbent } \\ \text { D. Barbano } & 2003 & \text { S. Moineau } \\ \text { C. H. White } & 2004 & \text { M. A. Drake } \\ \text { M. E. Johnson } & 2005 & \text { J. Lucey }\end{array}$

Continued 
Table 3 (Continued). American Dairy Science Association awards, 1981-2005

Foundation of International Association of Food Industry Suppliers Graduate Research Fellowship (1994)

$\begin{array}{llll}1994 & \text { E. J. Cole } & 2000 & \text { A. L. Harris } \\ 1996 & \text { T. J. Knight } & 2002 & \text { P. E. Groleau } \\ 1998 & \text { D. M. Norton } & 2004 & \text { D. Doucet }\end{array}$

International Dairy Foods Association Research Award in Dairy Foods Processing (1993)

$\begin{array}{llll}1993 & \text { E. R. Vedamuthu } & 1999 & \text { C. L. Hicks } \\ 1994 & \text { H. W. Modler } & 2000 & \text { D. M. Barbano } \\ 1995 & \text { R. W. Hartel } & 2001 & \text { S. S. Rizvi } \\ 1996 & \text { P. Jelen } & 2002 & \text { D. Dalgleish } \\ 1997 & \text { V. H. Holsinger } & 2003 & \text { S. Gunasekaran } \\ 1998 & \text { S. E. Gilliland } & 2004 & \text { V. Mistry }\end{array}$

International Dairy Production Award ${ }^{2}$ (1988)

\begin{tabular}{llll}
1988 & R. E. McDowell & 1997 & K. Sejrsen \\
1989 & C. Devendra & 1998 & L. R. McDowel \\
1990 & D. G. Armstrong & 1999 & R. W. Blake \\
1991 & K. L. Macmillan & 2000 & K. Agyemang \\
1992 & P. J. Van Soest & 2001 & C. P. Burvenich \\
1993 & R. D. Politiek & 2002 & M. A. Wattiaux \\
1994 & E. R. Orskov & 2003 & M. E. Goddard \\
1995 & V. Pedroso de Faria & 2004 & A. van Vuuren \\
1996 & J. Philipsson & 2005 & K. Togashi \\
\multicolumn{4}{c}{ J. L. Lush Award } \\
& \multicolumn{2}{c}{$(\mathbf{1 9 8 2})$} \\
1982 & C. R. Henderson & 1994 & R. E. Pearson \\
1983 & L. D. Van Vleck & 1995 & H. D. Norman \\
1984 & A. E. Freeman & 1996 & G. R. Wiggans \\
1985 & B. T. McDaniel & 1997 & R. L. Powell \\
1986 & W. E. Vinson & 1998 & M. Dentine \\
1987 & E. B. Burnside & 1999 & M. Soller \\
1988 & L. R. Schaefer & 2000 & P. VanRaden \\
1989 & D. Gianola & 2001 & G. E. Shook \\
1990 & B. W. Kennedy & 2002 & I. Mistal \\
1991 & R. L. Quaas & 2003 & B. G. Cassell \\
1992 & F. R. Allaire & 2004 & J. Dekkers \\
1993 & C. S. Smith & 2005 & J. F. Medrano
\end{tabular}

Kraft Foods and Milk Industry Foundation Teaching Award in Dairy Manufacturing (1956)

1981

1982

1983

1984

1985

1986

1987

1988

1989

1990

1991

1992

1993
R. L. Bradley

W. F. Shipe Jr.

1994

S. W. Seas

C. A. Ernstrom

F. W. Bodyfelt

J. T. Cardwell

K. R. Spurgeon

E. H. Marth

D. H. Kleyn

A. P. Hansen

R. L. Richter

D. E. Smith

C. H. White

Land O'Lakes, Inc. (2002)

1981

1982

1983

1984

1985

1986

1987

1988

1989

1990

1991

1992

1993
(Formerly Borden Inc. 1937-1997; Dean Foods 1998-2001)

$\begin{array}{lcll}\text { rly Borden Inc. 1937-1997; Dean Foods 1998-2001) } & 1986 \\ \text { T. Richardson } & 1994 & \text { D. B. Emmons } & 1987 \\ \text { A. E. Freeman } & 1995 & \text { M. J. Paape } & 1988 \\ \text { C. L. Davis } & 1996 & \text { T. R. Klaenhammer } & 1989 \\ \text { J. T. Huber } & 1997 & \text { W. E. Sandine } & 1990 \\ \text { H. M. Farrell } & 1998 & \text { J. H. Clark } & 1991 \\ \text { E. M. Marth } & 1999 & \text { S. C. Nickerson } & 1992 \\ \text { H. E. Swaisgood } & 2000 & \text { R. L. Horst } & 1993 \\ \text { N. F. Olson } & 2001 & \text { I. H. Mather } & \\ \text { R. S. Emery } & 2002 & \text { S. P. Oliver } & \\ \text { L. L. McKay } & 2003 & \text { D. J. Schingoethe } & \\ \text { R. W. Hemken } & 2004 & \text { K. L. Smith } & \\ \text { W. W. Thatcher } & 2005 & \text { L. Satter } & \\ \text { R. } & & & \end{array}$

\section{J. G. Parsons}

Ghristen

R. J. Baer

P. Jelen

J. Partridge

S. E. Gilliland

D. R. Henning

C. L. Hicks

R. Jiménez-Flores 1981

1982

1983

1984

1985

1986

1987

990

992
Table 3 (Continued). American Dairy Science Association awards, $1981-2005^{1}$

Land O'Lakes, Purina Feed, LLC Teaching Award in Dairy Production (2004)

(Formerly Purina Mills, Inc. 1973-2003)

$\begin{array}{lll}\text { D. A. Wieckert } & 1994 & \text { M. D. Kenealy } \\ \text { J. M. Elliot } & 1995 & \text { J. K. Hillers }\end{array}$

$\begin{array}{lll}\text { P. R. Shellenberger } & 1996 & \text { D. P. Dickson }\end{array}$

$\begin{array}{lll}\text { D. R. Mertens } & 1997 & \text { J. A. Gilmore }\end{array}$

$\begin{array}{lll}\text { L. D. Muller } & 1998 & \text { A. G. Hunter }\end{array}$

C. L. Norton $1999 \quad$ J. E. Pollak

D. M. Galton $2000 \quad$ E. J. DePeters

J. W. Fuquay $2001 \quad$ J. H. Herbein

J. F. Dickey $2002 \quad$ G. E. Shook

$\begin{array}{lll}\text { C. L. Moore } & 2003 & \text { R. S. Kensinger }\end{array}$

M. A. Barnes $2004 \quad$ W. Hurley

L. B. Hansen $2005 \quad$ J. Wohlt

E. H. Jaster

Danisco International Dairy Science Award (2005)

(Formerly Marschall Rhodia International Dairy Science Award 1981-2004)

$\begin{array}{lll}\text { T. A. J. Payens } & 1994 & \text { C. Daly } \\ \text { J. J. Stadhouders } & 1995 & \text { A. Jarvis } \\ \text { R. C. Lawrence } & 1996 & \text { J. N. de Wit } \\ \text { L. K. Creamer } & 1997 & \text { W. M. de Vos } \\ \text { W. Buchheim } & 1998 & \text { M. A. El Soda } \\ \text { G. Cheeseman } & 1999 & \text { A. Hillier } \\ \text { P. F. Fox } & 2000 & \text { V. L. Crow } \\ \text { B. Ribadeau-Dumas } & 2001 & \text { H. Singh } \\ \text { P. Walstra } & 2002 & \text { D. Horne } \\ \text { J.-L. Maubois } & 2003 & \text { N. P. Shah } \\ \text { M. Caric } & 2004 & \text { P. McSweeney } \\ \text { G. Venema } & 2005 & \text { P. A. Munro }\end{array}$

E. Renner

Merial Dairy Management Research Award (1998)

(Formerly the Merck AgVet Award)

$\begin{array}{lll}\text { J. L. Albright } & 1996 & \text { H. H. Van Horn } \\ \text { S. L. Spahr } & 1997 & \text { R. L. Nebel } \\ \text { L. D. Muller } & 1998 & \text { S. P. Oliver } \\ \text { M. L. McGilliard } & 1999 & \text { J. D. Quigley } \\ \text { G. H. Schmidt } & 2000 & \text { Not awarded } \\ \text { R. D. Appleman } & 2001 & \text { D. K. Beede } \\ \text { K. L. Smith } & 2002 & \text { W. W. Thatcher } \\ \text { P. A. Oltenacu } & 2003 & \text { L. K. Fox } \\ \text { B. G. Cassell } & 2004 & \text { G. Dahl } \\ \text { M. A. DeLorenzo } & 2005 & \text { R. Palmer }\end{array}$

National Milk Producers Federation

Richard M. Hoyt Memorial Award (1971)

$\begin{array}{lll}\text { A. Kuipers } & 1994 & \text { D. Romagnolo } \\ \text { L. Kung Jr. } & 1995 & \text { M. A. McGuire } \\ \text { R. S. Applebaum } & 1996 & \text { J. R. Pursley } \\ \text { M. E. Sanders } & 1997 & \text { C. R. West } \\ \text { B. A. Crooker } & 1998 & \text { T. R. Overton } \\ \text { P. M. Van Raden } & 1999 & \text { B. E. Dias } \\ \text { J. L. Vicini } & 2000 & \text { T. Mackle } \\ \text { E. T. Ryser } & 2001 & \text { R. P. Radcliff } \\ \text { D. P. Casper } & 2002 & \text { L. H. Baumgard } \\ \text { M. C. Lucy } & 2003 & \text { M. Oba } \\ \text { C. M. Cowan } & 2004 & \text { G. Bobe } \\ \text { D. A. Romero } & 2005 & \text { J. M. DeFrain } \\ \text { R. D. Byrne } & & \end{array}$

Continued 
Table 3 (Continued). American Dairy Science Association awards, $1981-2005^{1}$

\begin{tabular}{|c|c|c|c|}
\hline \multicolumn{4}{|c|}{$\begin{array}{c}\text { Nutrition Professionals, Inc. Applied Dairy } \\
\text { Nutrition Award (1991) }\end{array}$} \\
\hline 1991 & J. T. Huber & 1999 & E. J. DePeters \\
\hline 1992 & M. F. Hutjens & 2000 & A. J. Heinrichs \\
\hline 1993 & D. K. Beede & 2001 & M. S. Allen \\
\hline 1994 & J. E. Nocek & 2002 & R. Shaver \\
\hline 1995 & D. R. Mertens & 2003 & J. L. Firkins \\
\hline 1996 & D. J. Schingoethe & 2004 & R. Grummer \\
\hline 1997 & P. T. Chandler & 2005 & J. Goff \\
\hline 1998 & C. R. Staples & & \\
\hline \multicolumn{4}{|c|}{$\begin{array}{c}\text { Pfizer Animal Health Physiology Award (2003) } \\
\text { (Formerly Pharmacia \& Upjohn Animal Health } \\
\text { Physiology Award 1981-2002) }\end{array}$} \\
\hline 1981 & W. W. Thatcher & 1994 & D. E. Bauman \\
\hline 1982 & J. H. Britt & 1995 & R. D. Bremel \\
\hline 1983 & H. A. Tucker & 1996 & F. C. Gwazdauskas \\
\hline 1984 & R. P. Amann & 1997 & P. J. Hansen \\
\hline 1985 & R. H. Foote & 1998 & M. E. Kehrli Jr. \\
\hline 1986 & G. E. Seidel Jr. & 1999 & G. B. Anderson \\
\hline 1987 & P. V. Malven & 2000 & R. M. Akers \\
\hline 1988 & R. G. Saacke & 2001 & M. Wiltbank \\
\hline 1989 & R. L. Horst & 2002 & J. S. Stevenson \\
\hline 1990 & R. L. Ax & 2003 & M. C. Lucy \\
\hline 1991 & R. J. Collier & 2004 & L. Spicer \\
\hline 1992 & T. A. Reinhardt & 2005 & A. Capuco \\
\hline 1993 & H. A. Garverick & & \\
\hline \multicolumn{4}{|c|}{ Pioneer Hi-Bred Forage Award (1991) } \\
\hline 1991 & D. R. Waldo & 1999 & L. Kung \\
\hline 1992 & G. C. Fahey Jr. & 2000 & L. O. Ely \\
\hline 1993 & P. J. Van Soest & 2001 & W. K. Coblentz \\
\hline 1994 & L. D. Satter & 2002 & Not awarded \\
\hline 1995 & M. S. Allen & 2003 & J. R. Russell \\
\hline 1996 & J. H. Harrison & 2004 & D. Mertens \\
\hline 1997 & H.-J. G. Jung & 2005 & K. A. Beauchemin \\
\hline 1998 & L. D. Muller & & \\
\hline \multicolumn{4}{|c|}{ West Agro, Inc. (1979) } \\
\hline 1981 & W. N. Philpot & 1994 & J. S. Hogan \\
\hline 1982 & R. P. Natzke & 1995 & M. E. Kehrli Jr. \\
\hline 1983 & W. D. Schultze & 1996 & A. J. Guidry \\
\hline 1984 & K. L. Smith & 1997 & W. L. Hurley \\
\hline 1985 & S. C. Nickerson & 1998 & L. M. Sordillo \\
\hline 1986 & R. J. Eberhart & 1999 & K. E. Leslie \\
\hline 1987 & D. E. Jasper & 2000 & R. Erskine \\
\hline 1988 & J. W. Pankey & 2001 & D. E. Morin \\
\hline 1989 & R. J. Harmon & 2002 & B. M. Jayarao \\
\hline 1990 & G. M. Jones & 2003 & Y. H. Schukken \\
\hline 1991 & L. K. Fox & 2004 & D. Kelton \\
\hline 1992 & S. P. Oliver & 2005 & L. Timms \\
\hline 1993 & R. J. Yancey & & \\
\hline \multicolumn{4}{|c|}{ Dairy Research Foundation (DRINC, Inc.) (1971) } \\
\hline 1981 & P. M. T. Hansen & 1986 & R. C. Lindsay \\
\hline 1982 & L. L. McKay & 1987 & S. E. Gilliland \\
\hline 1983 & K. M. Shahani & 1988 & H. W. Modler \\
\hline 1984 & G. H. Richardson & 1989 & V. R. Harwalkar \\
\hline 1985 & H. E. Swaisgood & 1990 & T. R. Klaenhammer \\
\hline \multicolumn{4}{|c|}{$\begin{array}{c}\text { National Dairy Herd Improvement } \\
\text { Association Award (1983) }\end{array}$} \\
\hline 1983 & F. N. Dickinson & 1987 & K. R. Butcher \\
\hline 1984 & B. H. Crandall & 1988 & D. C. Jordan \\
\hline 1985 & D. E. Voelker & 1989 & W. M. Dillon \\
\hline 1986 & C. C. Olson & & \\
\hline
\end{tabular}

Continued
Table 3 (Continued). American Dairy Science Association awards, 1981-2005

\begin{tabular}{llcl}
\hline \multicolumn{5}{c}{ American Cyanamid Award (1986) } \\
1986 & S. B. Spencer & 1990 & D. E. Otterby \\
1987 & D. E. Bauman & 1991 & J. H. Clark \\
1988 & L. D. Satter & 1992 & D. C. Beitz \\
1989 & D. L. Palmquist & 1993 & I. H. Mather \\
\multicolumn{4}{c}{ FASS-American Feed Industry Association } \\
New Frontiers in Animal Nutrition Award (2004) \\
2004 & D. E. Bauman \\
2005 & J. Clark & & \\
\multicolumn{4}{c}{ Award of Honor (1959) } \\
1981 & R. P. Niedermeier & 1994 & H. H. Van Horn \\
1982 & W. L. Dunkley & 1995 & J. H. Martin \\
1983 & J. K. Loosli & 1996 & R. T. Marshall \\
1984 & T. H. Blosser & 1997 & N. F. Olson \\
1985 & E. N. Boyd & 1998 & R. W. Hemken \\
1986 & L. D. McGilliard & 1999 & L. D. Satter \\
1987 & J. R. Campbell & 2000 & J. H. Clark \\
1988 & L. H. Schultz & 2001 & R. P. Natzke \\
1989 & J. E. Legates & 2002 & J. W. Fuquay \\
1990 & V. H. Nielsen & 2003 & S. Gilliland \\
1991 & D. L. Bath & 2004 & N. Jorgensen \\
1992 & R. L. Sellars & 2005 & L. Muller \\
1993 & B. R. Baumgardt & &
\end{tabular}

Distinguished Service Award (1961)

\begin{tabular}{llll}
1981 & G. W. Reinbold & 1994 & R. D. Plowman \\
1982 & E. Lundstedt & 1995 & N. A. Jorgensen \\
1983 & W. P. Flatt & 1996 & T. W. Holzinger \\
1984 & J. H. Erb & 1997 & G. A. Muck \\
1985 & K. G. Savage & 1998 & A. E. Freeman \\
1986 & R. Jenness & 1999 & S. Patton \\
1987 & J. W. Crowley & 2000 & J. Lauderdale \\
1988 & W. C. McGinnis & 2001 & D. H. Hettinga \\
1989 & N. L. Jacobson & 2002 & J. H. Clark \\
1990 & C. A. Ernstrom & 2003 & B. Baumgardt \\
1991 & R. E. Walton & 2004 & S. Larson \\
1992 & L. S. Mix & 2005 & J. Young \\
1993 & J. P. Jordan & & \\
\hline
\end{tabular}

${ }^{1}$ The Genevieve Christen Undergraduate Student Award winners are noted later (Table 19).

${ }^{2}$ Sponsored by Ralston Purina International from 1988-1999 and by Elanco Animal Health from 2000 to the present.

${ }^{3}$ Sponsored by ABS Global, Inc.

the Journal of Dairy Science or pay for hard copy. All those members of ADSA who became Life Members during the last $25 \mathrm{yr}$ are noted in Table 5 . Thank you to each of you for your long-term interest in and support of our ADSA!

\section{PURINA MILLS RESEARCH FELLOWSHIPS}

In 1948, C. A. Elvehjem (Association of Land Grant Colleges), R. M. Bethke (Poultry Science Association, PSA), K. L. Turk (ADSA), M. A. Emmerson (American Veterinary Medical Association), W. M. Beeson (ASAS), H. L. Wilcke (Purina Mills, Inc.), and E. B. Powell (Purina Mills, Inc.) culminated their previous work with the 
Table 4. Fellows of American Dairy Science Association

\begin{tabular}{lllll}
\hline $\mathbf{1 9 9 7}$ (Inducted) & $\mathbf{1 9 9 7}$ (Inducted) & $\mathbf{1 9 9 8}$ & $\mathbf{2 0 0 1}$ & $\mathbf{2 0 0 4}$ \\
D. L. Bath & J. H. Martin & J. O. Almquist & D. C. Beitz & J. C. Bruhn \\
B. R. Baumgardt & L. D. McGilliard & J. M. Elliott & R. H. Foote & T. Klaenhammer \\
T. H. Blosser & F. E. Nelson & A. E. Freeman & J. W. Fuquay & L. L. McKay \\
E. N. Boyd & V. H. Nielsen & S. E. Gilliland & H. D. Norman & L. Muller \\
J. R. Campbell & W. M. Roberts & E. H. Marth & C. E. Polan & M. J. Paape \\
B. H. Crandall & L. H. Schultz & N. F. Olson & H. A. Tucker & 2005 \\
W. L. Dunkley & R. L. Sellars & $\mathbf{1 9 9 9}$ & $\mathbf{2 0 0 2}$ & W. L. Crist \\
C. A. Ernstrom & H. H. Van Horn & J. Brunner & R. L. Bradley, Jr. & M. D. Kenealy \\
W. P. Flatt & G. W. Weigold & H. R. Conrad & J. K. Hillers & L. R. McDowell \\
L. G. Harmon & $\mathbf{1 9 9 7}$ Elected) & W. J. Harper & P. G. Keeney & R. L. Powell \\
H. B. Henderson & J. A. Albright & R. W. Hemken & G. H. Richardson & G. E. Shook \\
T. W. Holzinger & R. L. Baldwin & T. W. Keenan & K. L. Smith & \\
N. L. Jacobson & R. S. Emery & J. W. Thomas & G. A. Somkuti & \\
R. Jenness & H. M. Farrell, Jr. & $\mathbf{2 0 0 0}$ & $\mathbf{2 0 0 3}$ & \\
N. A. Jorgensen & J. T. Huber & D. B. Emmons & J. H. Clark & \\
D. B. Josephson & S. Patton & R. G. Jensen & D. L. Palmquist & \\
W. D. Knox & & L. D. Satter & W. E. Sandine & \\
J. E. Legates & & S. B. Spencer & & \\
J. K. Loosli & & H. E. Swaisgood & & \\
R. T. Marshall & & J. W. Young & & \\
\hline
\end{tabular}

establishment of the Purina Mills Research Fellowship. Fellowships were to be granted in 2 categories of animal science-the fields of nutritional research as applied to dairy, poultry, and animal husbandry and to research in transmissible disease of livestock and poultry. Awarding Fellowships to graduate students in veterinary medicine occurred for only a few years. Fellowships were awarded based on academic qualifications and scientific merit of the graduate student applicants. Recipients were selected by an independent committee of professors and scientists appointed by ADSA, ASAS, and PSA. The purpose of the program was to assist in the graduate training of personnel for leadership in the nutritional sciences of food and companion animals. Awards were awarded annually for up to $3 \mathrm{yr}$ of graduate study in an accredited graduate program at a US or Canadian university. Later in the program, one individual could hold the Fellowship for only $1 \mathrm{yr}$ to provide the honor to a larger number of graduate students. Initially, the dollar value of the Fellowship was $\$ 1,440$. The stipend for each of the last offered Fellowships was $\$ 12,500$. As one peruses the list of Purina Mills Fellows in Table 6, it can be seen that the goal of training leaders in animal agriculture was met by the program. Iowa State University, Oklahoma State University, Purdue University, University of California-Davis, and University of Illinois were the 5 universities that trained the greatest number of Purina Mills Fellows. During the tenure of the program, Fellows chose at least 36 different US institutions and at least 4 different Canadian universities. Furthermore, 25 of the 230 Fellows held the Fellowships for 3 yr. The ADSA and the other relevant animal agriculture societies have much reason to sincerely thank Purina Mills for its long-time
(52 yr) and generous financial support of this Fellowship program. No requirements were placed on the Fellows by Purina Mills. Clearly, Purina Mills Research Fellowships were regarded very highly within the animal scientific community.

\section{LEADERSHIP OF ADSA}

During the 25-yr period from 1981 to the present time, the ADSA has been fortunate to be led by outstanding elected officers, committee chairs and members, and salaried staff members. The board of directors consisted of president, vice president, treasurer (appointed by board), executive director (formerly executive secretary), editor-in-chief, past president, and 6 directors. The board of directors operated with 2 internal committees-the 6-member Executive committee composed of the president, vice president, past president, editor-in-chief, executive director, and treasurer and the 4-member Internal Affairs committee composed of the vice president (chair), 2 senior directors, and the immediate past director from the division other than that of vice president. The Dairy Foods and Production Divisions each elect a new director to the board each year for a 3-yr term. At the beginning of this 25-yr period, an assistant executive secretary and associate editor also participated in board meetings. A listing of the presidents during each year from 1981 through 2006 is in Table 7. Treasurers and historians are listed in Table 8, executive secretaries/directors are in Table 9, and directors are in Table 10. The Dairy Foods and the Production divisions each provide a newly elected vice president every other year who then becomes president the following year. Twelve of the presidents during 
the previous $25 \mathrm{yr}$ were from the Midwest region of the United States, 7 were from the Southeast, 5 were from the West, and 1 was from the Northeast. Five presidents were from the state of Wisconsin, and 3 were from California and Illinois. In respect to our presidential leaders, their pictures are presented in Figure 1. During the 100-yr history of ADSA, 9 presidents were from New York and Wisconsin, 8 were from Michigan, 7 were from Illinois, and 6 were from California (Table 11). Twenty-six other states provided presidents to lead the ADSA. Standing committees of the board of directors at the end of the 25-yr period were the Nominating, Membership, Finance, Awards, Journal Management, Program, and Resolutions committees. The International Relations committee became a Special committee. With regard to the Special committees, the position

Table 5. Life membership, 1981-2005

\begin{tabular}{|c|c|c|c|}
\hline 1981 & 1982 & 1983 & 1984 \\
\hline $\begin{array}{l}\text { H. W. Anderson } \\
\text { W. Carter } \\
\text { M. L. Speck } \\
\text { J. D. Bowers } \\
\text { H. A. Hollender } \\
\text { A. B. Storrs }\end{array}$ & $\begin{array}{l}\text { G. K. Davis } \\
\text { C. Harper Jr. } \\
\text { M. Loewenstein } \\
\text { F. I. Elliott } \\
\text { J. H. Hetrick } \\
\text { L. D. McGilliard } \\
\text { E. L. Thomas } \\
\text { L. O. Ely } \\
\text { J. G. Laeder } \\
\text { F. R. Murdock } \\
\text { K. E. Harshbarger }\end{array}$ & $\begin{array}{l}\text { J. O. Almquist } \\
\text { R. Y. Cannon } \\
\text { C. L. Hankinson } \\
\text { G. E. Hawkins } \\
\text { R. I. Meyer } \\
\text { J. P. Mixner } \\
\text { C. L. Pelissier } \\
\text { F. G. Warren }\end{array}$ & $\begin{array}{l}\text { D. C. Church } \\
\text { N. Eisenstein } \\
\text { F. E. Elliott } \\
\text { W. S. Gaunya } \\
\text { E. C. Hagberg } \\
\text { J. W. Hibbs } \\
\text { J. R. Kilgore } \\
\text { T. Kristoffersen } \\
\text { W. M. Menz } \\
\text { R. W. Wayne }\end{array}$ \\
\hline 1985 & 1986 & 1987 & 1988 \\
\hline $\begin{array}{l}\text { B. R. Baker } \\
\text { A. L. Brundage } \\
\text { M. Burger } \\
\text { G. A. Damisch Jr. } \\
\text { N. W. Hooven Jr. } \\
\text { R. Jenness } \\
\text { J. J. Jezeski } \\
\text { F. M. Johnson } \\
\text { R. W. Kunkel } \\
\text { J. K. Loosli } \\
\text { J. W. Lusk } \\
\text { F. H. McDowell } \\
\text { R. P. Niedermeier } \\
\text { C. L. Norton } \\
\text { G. J. Smith } \\
\text { G. W. Weigold } \\
\text { L. S. Wittwer }\end{array}$ & $\begin{array}{l}\text { R. J. Baker } \\
\text { R. Bassett } \\
\text { H. E. Bechtel } \\
\text { L. F. Blanton } \\
\text { A. R. Brazis } \\
\text { M. Burger } \\
\text { W. H. Burkitt } \\
\text { C. H. Burton } \\
\text { E. W. Custer } \\
\text { C. G. M. Edgerly } \\
\text { P. R. Elliker } \\
\text { W. K. Fox }\end{array}$ & $\begin{array}{l}\text { V. R. Bohman } \\
\text { R. H. Bundus } \\
\text { F. A. Duarte } \\
\text { C. F. Foreman } \\
\text { D. M. Graham } \\
\text { T. I. Hedrick } \\
\text { W. K. Jordan } \\
\text { E. T. Oleski } \\
\text { C. R. Richards } \\
\text { O. T. Stallcup } \\
\text { G. E. Stoddard } \\
\text { D. E. Voelker } \\
\text { J. O. Young }\end{array}$ & $\begin{array}{l}\text { K. P. Anderson } \\
\text { E. W. Bird } \\
\text { T. H. Blosser } \\
\text { M. P. Bryant } \\
\text { L. J. Bush } \\
\text { J. C. Flake } \\
\text { J. H. Koshi } \\
\text { K. W. Royer } \\
\text { R. W. Stanley } \\
\text { J. R. Staubus } \\
\text { H. Voelker } \\
\text { B. H. Webb }\end{array}$ \\
\hline 1989 & 1990 & 1991 & 1992 \\
\hline $\begin{array}{l}\text { F. N. Andrews } \\
\text { H. J. Bearden } \\
\text { E. N. Boyd } \\
\text { F. Eldridge } \\
\text { R. J. Flipse } \\
\text { C. T. Herald } \\
\text { A. S. Hodgson } \\
\text { B. C. Johnson } \\
\text { F. W. Lengemann } \\
\text { R. M. Luther } \\
\text { R. E. Mather } \\
\text { R. B. Maxcy } \\
\text { E. M. Mikolajcik } \\
\text { R. D. Mochrie } \\
\text { S. D. Musgrave } \\
\text { A. L. Rippen } \\
\text { B. A. Webb } \\
\text { C. W. Yeager Jr. }\end{array}$ & $\begin{array}{l}\text { K. M. Barth } \\
\text { H. J. Bearden } \\
\text { H. W. Colvin Jr. } \\
\text { J. M. deMan } \\
\text { A. H. Duthie } \\
\text { M. G. Farnham } \\
\text { D. J. Hankinson } \\
\text { W. J. Harper } \\
\text { E. S. Humbert } \\
\text { N. L. Jacobson } \\
\text { W. M. Miller } \\
\text { G. K. Murthy } \\
\text { D. Olds } \\
\text { H. H. Olson } \\
\text { C. C. Olson } \\
\text { W. G. Riemann } \\
\text { O. M. Russell } \\
\text { G. E. Stoddard } \\
\text { G. M. Ward } \\
\text { V. L. Zehren } \\
\text { H. P. Zimmerman }\end{array}$ & $\begin{array}{l}\text { D. R. Frazem } \\
\text { H. D. Johnson } \\
\text { M. E. Morgan }\end{array}$ & $\begin{array}{l}\text { W. J. Corbett } \\
\text { J. L. Evans } \\
\text { H. L. Mitten } \\
\text { J. H. Nelson } \\
\text { V. S. Packard } \\
\text { J. C. Rennie } \\
\text { W. E. Sandine } \\
\text { D. B. Stussi } \\
\text { H. Timmen } \\
\text { N. Vandemark } \\
\text { R. G. Warner } \\
\text { R. R. Zall }\end{array}$ \\
\hline
\end{tabular}


Table 5 (Continued). Life membership, 1981-2005

\begin{tabular}{|c|c|c|c|}
\hline 1993 & 1994 & 1995 & 1996 \\
\hline $\begin{array}{l}\text { V. G. Anderson } \\
\text { L. J. Boyd } \\
\text { F. M. Crane } \\
\text { V. A. Jones } \\
\text { C. M. Lawrence } \\
\text { J. B. Lindamood } \\
\text { G. K. MacLeod } \\
\text { F. G. Owen } \\
\text { J. Prow } \\
\text { G. H. Richardson } \\
\text { G. C. Scott } \\
\text { J. W. Smith } \\
\text { J. H. Vandersall }\end{array}$ & $\begin{array}{l}\text { D. L. Bath } \\
\text { W. H. Brown } \\
\text { A. C. Linnerud } \\
\text { C. L. Moore } \\
\text { S. H. Morrison } \\
\text { F. E. Pardue } \\
\text { D. E. Pritchard } \\
\text { J. M. Rakes } \\
\text { H. A. Ramsey } \\
\text { C. W. Richardson } \\
\text { Z. Saito } \\
\text { J. R. Staubus } \\
\text { R. L. Steele } \\
\text { G. A. Stewart } \\
\text { H. L. Wildesin }\end{array}$ & $\begin{array}{l}\text { S. Arima } \\
\text { F. M. Crane } \\
\text { J. F. Dickey } \\
\text { W. M. Dillon } \\
\text { T. G. Martin } \\
\text { R. E. McDowell } \\
\text { K. Shahani } \\
\text { E. Ueyama }\end{array}$ & $\begin{array}{l}\text { W. B. Anthony } \\
\text { C. A. Becker } \\
\text { S. B. Carr } \\
\text { E. G. Carrancedo } \\
\text { R. B. Eckberg } \\
\text { E. A. Guene } \\
\text { W. H. Gossett } \\
\text { H. D. Graham } \\
\text { W. C. Jacobson } \\
\text { R. H. Miller } \\
\text { F. E. Nelson } \\
\text { A. H. Rakes } \\
\text { G. H. Schmidt } \\
\text { J. W. Sherbon } \\
\text { W. Taylor } \\
\text { P. J. Van Soest } \\
\text { C. J. Wilcox }\end{array}$ \\
\hline 1997 & 1998 & 1999 & 2000 \\
\hline $\begin{array}{l}\text { M. W. Colburn } \\
\text { G. S. Hess } \\
\text { T. W. Holzinger } \\
\text { J. C. Johnson, Jr. } \\
\text { A. J. Lee } \\
\text { J. H. Martin } \\
\text { C. H. Noller } \\
\text { L. M. Schugel } \\
\text { P. R. Shellenberger } \\
\text { P. M. Smith } \\
\text { L. W. Specht } \\
\text { D. A. Wieckert }\end{array}$ & $\begin{array}{l}\text { C. W. Arave } \\
\text { D. K. Bandler } \\
\text { S. E. Barnard } \\
\text { D. L. Ingle } \\
\text { R. C. Lamb } \\
\text { J. E. Moore } \\
\text { R. L. Park } \\
\text { P. A. Putman } \\
\text { A. A. Rosales } \\
\text { R. L. Sellars } \\
\text { C. B. Theuer } \\
\text { R. L. Vetter } \\
\text { T. Wallace }\end{array}$ & $\begin{array}{l}\text { R. S. Adams } \\
\text { D. G. Braund } \\
\text { G. J. Brisson } \\
\text { W. L. Dunkley } \\
\text { R. H. Foote } \\
\text { J. W. Fuquay } \\
\text { J. B. Holter } \\
\text { E. L. Jensen } \\
\text { W. E. Sandine }\end{array}$ & $\begin{array}{l}\text { J. L. Albright } \\
\text { D. V. Armstrong } \\
\text { E. V. Caruolo } \\
\text { D. B. Emmons } \\
\text { W. P. Flatt } \\
\text { S. H. Gretebeck } \\
\text { D. Hillman } \\
\text { M. P. Kalmbach } \\
\text { I. Kiyosawa } \\
\text { W. S. LaGrange } \\
\text { L. M. Larsen } \\
\text { C. Macgregor } \\
\text { J. K. Miller } \\
\text { J. L. Morrill } \\
\text { J. R. Seoane } \\
\text { T. W. White }\end{array}$ \\
\hline 2001 & 2002 & 2003 & 2004 \\
\hline $\begin{array}{l}\text { C. B. Ammerman } \\
\text { A. N. Bringe } \\
\text { J. V. Chambers } \\
\text { P. T. Chandler } \\
\text { J. K. Hillers } \\
\text { P. V. Malven } \\
\text { M. J. Montgomery } \\
\text { T. Nagasawa } \\
\text { R. L. Selman } \\
\text { H. E. Swaisgood } \\
\text { L. Swanson } \\
\text { J. W. Young }\end{array}$ & $\begin{array}{l}\text { A. Bezkorovainy } \\
\text { R. L. Bradley Jr. } \\
\text { G. F. W. Haenlein } \\
\text { V. W. Hays } \\
\text { V. G. Janolino } \\
\text { T. W. Keenan } \\
\text { P. G. Keeney } \\
\text { R. C. Laben } \\
\text { G. A. Muck } \\
\text { J. G. Parsons } \\
\text { C. E. Polan } \\
\text { J. L. Robinson } \\
\text { H. H. Van Horn Jr. } \\
\text { D. R. Waldo } \\
\text { J. M. White }\end{array}$ & $\begin{array}{l}\text { D. G. Britt } \\
\text { A. E. Freeman } \\
\text { R. V. Josephson } \\
\text { C. J. Kercher } \\
\text { D. S. Kronfeld } \\
\text { R. G. Saacke } \\
\text { W. G. Schmutz } \\
\text { K. L. Simkins Jr. } \\
\text { C. J. Sniffen } \\
\text { D. T. Vines }\end{array}$ & $\begin{array}{l}\text { F. D. Bisplinghoff } \\
\text { T. Burnside } \\
\text { M. Grossman } \\
\text { H. H. Head } \\
\text { L. F. Krabill } \\
\text { D. K. Nelson } \\
\text { G. Marx } \\
\text { R. T. Marshall } \\
\text { B. T. McDaniel } \\
\text { B. R. Moss } \\
\text { L. D. Muller } \\
\text { J. S. Norwood } \\
\text { R. L. Olson } \\
\text { L. D. Satter }\end{array}$ \\
\hline \multicolumn{4}{|l|}{2005} \\
\hline $\begin{array}{l}\text { J. H. Clark } \\
\text { G. McCoy } \\
\text { B. T. McDaniel } \\
\text { W. A. Olson } \\
\text { H. F. Tyrrell }\end{array}$ & & & \\
\hline
\end{tabular}

of historian was continued and the Graduate Student Paper Contest committees were transferred to each of the Councils for the Dairy Foods and Production divi- sions during the 25-yr period. During the same period, these Special committees were established: ADSA Foundation Trustees, FASS, Centennial Oversight/ 
Table 6. Purina Mills Fellows throughout the history of the program ${ }^{1}$

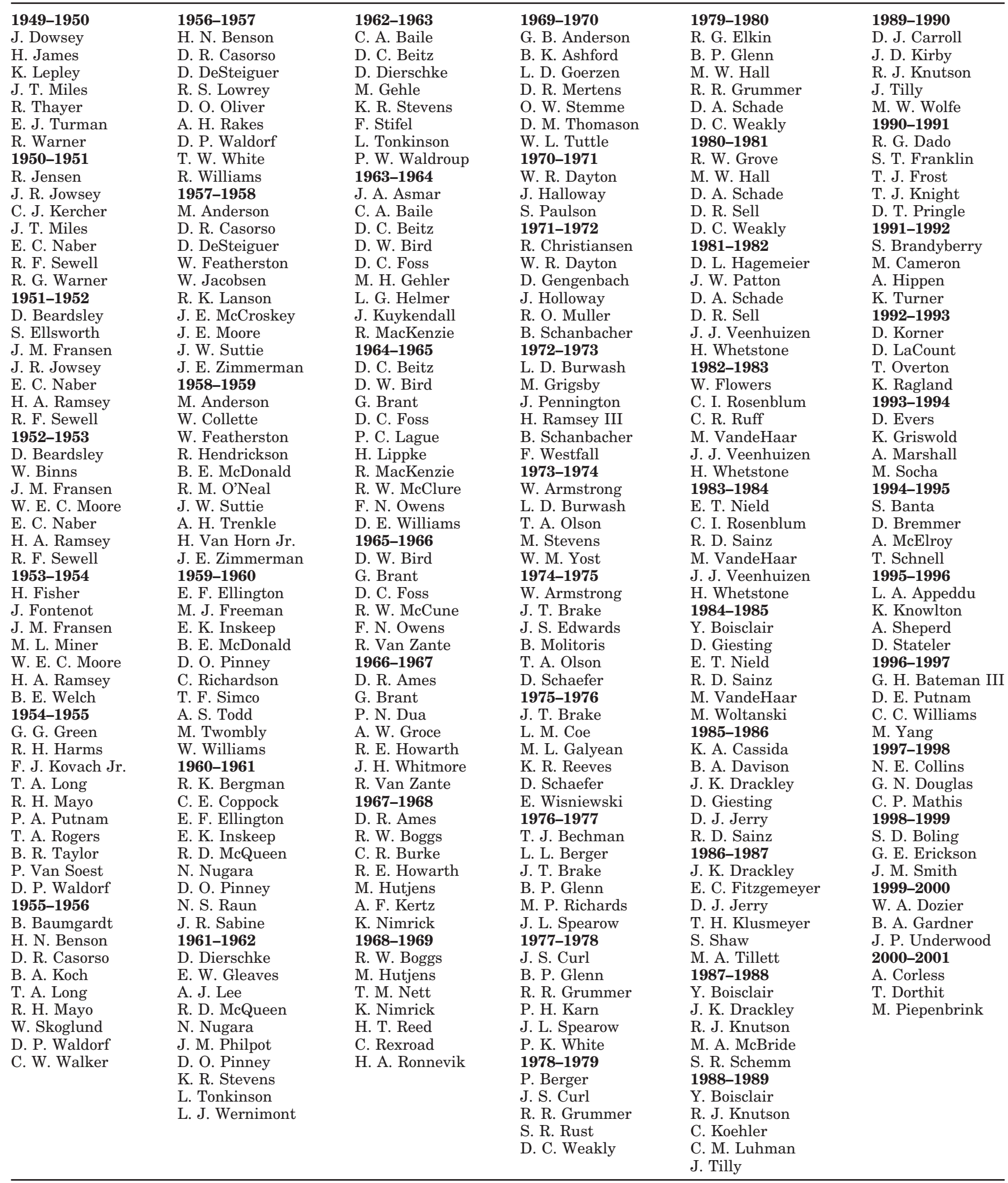

\footnotetext{
${ }^{1}$ The listing begins in 1949 because the names of fellows before 1981 have not been published previously.
} 
Table 7. Presidents of the American Dairy Science Association, 19812005

\begin{tabular}{lll}
\hline Year & President & University \\
\hline 1981 & J. R. Campbell & Illinois \\
1982 & J. H. Martin & Clemson \\
1983 & L. H. Schultz & Wisconsin \\
1984 & R. T. Marshall & Missouri \\
1985 & B. R. Baumgardt & Purdue \\
1986 & N. F. Olson & Wisconsin \\
1987 & D. L. Bath & California \\
1988 & G. H. Richardson & Utah \\
1989 & H. H. Van Horn & Florida \\
1990 & R. L. Sellars & Chr. Hansen Lab, Inc., Wisconsin ${ }^{1}$ \\
1991 & N. A. Jorgensen & Wisconsin \\
1992 & S. E. Gilliland & Oklahoma State \\
1993 & J. H. Clark & Illinois \\
1994 & W. E. Sandine & Oregon State \\
1995 & R. W. Hemken & Kentucky \\
1996 & R. L. Richter & Texas A \& M \\
1997 & L. D. Satter & Wisconsin \\
1998 & C. H. White & Mississippi State \\
1999 & L. D. Muller & Pennsylvania State \\
2000 & H. E. Swaisgood & North Carolina State \\
2001 & D. J. Schingoethe & South Dakota State \\
2002 & J. C. Bruhn & California-Davis \\
2003 & D. K. Beede & Michigan State \\
2004 & J. A. O’Donnell & California \\
2005 & M. F. Hutjens & Illinois \\
\hline
\end{tabular}

${ }^{1}$ Industry/commodity groups.

Task Force, Centennial Planning and Budget, Education, and Research.

The Dairy Foods and Production Divisions of the ADSA were led by members elected as chair, vice chair,

Table 8. Treasurers and historians of the American Dairy Science Association, 1980-2005

\begin{tabular}{lll}
\hline Year & Treasurer & Historian \\
\hline $1980-1981$ & T. H. Blosser & G. M. Trout \\
$1981-1982$ & T. H. Blosser & G. M. Trout \\
$1982-1983$ & T. H. Blosser & L. G. Harmon \\
$1983-1984$ & J. R. Campbell & L. G. Harmon \\
$1984-1985$ & J. R. Campbell & L. G. Harmon \\
$1985-1986$ & J. R. Campbell & L. G. Harmon \\
$1986-1987$ & L. H. Schultz & W. L. Dunkley \\
$1987-1988$ & L. H. Schultz & W. L. Dunkley \\
$1988-1989$ & L. H. Schultz & W. L. Dunkley \\
$1989-1990$ & D. L. Bath & T. H. Blosser \\
$1990-1991$ & D. L. Bath & T. H. Blosser \\
$1991-1992$ & D. L. Bath & T. H. Blosser \\
$1992-1993$ & R. T. Marshall & T. H. Blosser \\
$1993-1994$ & R. T. Marshall & T. H. Blosser \\
$1994-1995$ & R. T. Marshall & J. Tobias \\
$1995-1996$ & R. L. Sellars & J. Tobias \\
$1996-1997$ & R. L. Sellars & J. Tobias \\
$1997-1998$ & R. L. Sellars & J. Tobias \\
$1998-1999$ & H. H. Van Horn & J. Tobias \\
$1999-2000$ & H. H. Van Horn & J. Tobias \\
$2000-2001$ & H. H. Van Horn & J. R. Campbell \\
$2001-2002$ & R. L. Richter & J. R. Campbell \\
$2002-2003$ & R. L. Richter & J. R. Campbell \\
$2003-2004$ & R. L. Richter & J. R. Campbell \\
$2004-2005$ & E. Jordan & J. R. Campbell \\
\hline
\end{tabular}

secretary, and director (member-at-large for Dairy Foods). The chairs of each Division during the previous 25 -yr period are named in Table 12. The Councils of the Dairy Foods and Production divisions were assisted by several standing committees developed for specific activities of each division.

The ADSA began the previous 25-yr period with 2 branches (Northeast and Southern) that held annual meetings consisting of scientific exchange, business meetings, and award presentations. Because of the efforts of Jeffrey Keown, a Midwest Branch was approved in 1990. The initial officers of the Midwest Branch were J. Keown, President, University of Nebraska; J. Linn, Vice President, University of Minnesota; and D. Schingoethe, Secretary/Treasurer, South Dakota State University. The Midwest Branch consists of scientists in Illinois, Indiana, Iowa, Kansas, Michigan, Minnesota, Missouri, Nebraska, North Dakota, Ohio, South Dakota, and Wisconsin. The Midwest Branch holds its annual meeting in Des Moines, IA, along with the Midwest Section of ASAS. The presidents of each branch during the previous $25 \mathrm{yr}$ are named in Table 13. The annual meetings of the branches clearly assist the ADSA in carrying out its mission by localizing the ADSA within a region of the country. Perhaps the next $25 \mathrm{yr}$ will see the development of a Western branch of the ADSA.

Special attention to the position of executive director is appropriate. Claude J. Cruse began his career with the ADSA as acting treasurer in 1964 and treasurer in 1965. In 1966, one of the most significant single decisions of the third 25-yr history of ADSA was the hiring by ADSA of C. J. Cruse as executive secretary. During his tenure until 1985 (Table 9), ADSA purchased its Clark Street office building in Champaign, IL, and began in-house publication of the Journal of Dairy Science and other agricultural journals. C. D. Johnson, executive director from 1986 to 1997, coordinated the computerization of the ADSA headquarters. Our current executive director, Brenda Carlson, continues the tradition of excellence in leadership in the ADSA headquarters.

\section{STUDENT AFFILIATE DIVISION}

The SAD of ADSA has shown a steady pace of development to its current status. As described by Trout (1964), the $\mathrm{SAD}$ could be said to have its origins in a suggestion by J. H. Frandsen, the 7th and 8th president of ADSA, who spoke favorably of a junior membership in the ADSA. Associate memberships for students were established in 1936, and 30 students became members by the end of 1937. Membership grew to 391 by the end of 1941 but decreased significantly during World War II; membership expanded during the post-war years to 
Table 9. Executive secretary/directors of the American Dairy Science Association, 1980-2005

\begin{tabular}{llll}
\hline Year & Name & Year & Name \\
\hline $1980-1981$ & C. J. Cruse & $1993-1994$ & C. D. Johnson \\
$1981-1982$ & C. J. Cruse & $1994-1995$ & C. D. Johnson \\
$1982-1983$ & C. J. Cruse & 1996 & C. D. Johnson \\
$1983-1984$ & C. J. Cruse & 1997 & C. D. Johnson \\
$1984-1985$ & C. J. Cruse & 1998 & C. Sapp (interim); M. E. Kelley \\
$1985-1986$ & R. A. Schmidt (interim) & $1998-1999$ & M. E. Kelley \\
$1986-1987$ & C. D. Johnson & $1999-2000$ & K. Williamson \\
$1987-1988$ & C. D. Johnson & $2000-2001$ & K. Williamson/B. S. Carlson \\
$1988-1989$ & C. D. Johnson & 2002 & B. S. Carlson \\
$1989-1990$ & C. D. Johnson & 2003 & B. S. Carlson \\
$1990-1991$ & C. D. Johnson & 2004 & B. S. Carlson \\
$1991-1992$ & C. D. Johnson & 2005 & B. S. Carlson \\
$1992-1993$ & C. D. Johnson & & \\
\hline
\end{tabular}

859 members in 1949. The first Student Affiliate was considered in 1937, and bylaws were established for an affiliate or local chapter membership in ADSA in 1943. Cornell University and Oklahoma State were the first affiliate members; one at Penn State University followed shortly. There were 16 student affiliates already in 1948 and 21 in 1952. Beginning in 1954, student affiliate exhibits embellished the national ADSA meetings. The National Student Branch (now SAD) was officially organized and approved in 1959, with the first official meeting occurring at the 55th annual meeting of ADSA in 1960. L. D. Meyer of Michigan served as its first president. J. A. Lineweaver of Virginia, N. Nichols of Oklahoma, R. Ricketts of Missouri, and J. Williams of Clemson followed in order.

In 2005 , the SAD consists of 45 student affiliate chapters across the United States. As guided by its constitution, the SAD continues its efforts (1) to be a communication channel for exchange of information among its member chapters and between the ADSA and member chapters, (2) to acquaint students with all facets of ADSA, and (3) to develop leadership and promote scholastic achievement among its student members. The SAD accomplishes its goals, in part, by holding annual meetings with the parent society and by recognizing the following:

- Outstanding Student Affiliate Chapter

- Outstanding Student Affiliate Member

- Outstanding Chapter Advisor

- Outstanding Yearbook and Scrapbook

- Undergraduate Paper Presentation Winners

- ADSA Scholarship Recognition

- Outstanding Chapter Website

Annual meetings of regional branches (Northeast, Southern, and Midwest) of SAD conduct similar activities and awards to accomplish the above-stated goals. Participation in regional and national activities is bene- ficial for both the local chapters and for individual members. The activities of SAD at the annual ADSA meeting culminate in the SAD awards luncheon in which the Awards are presented and newly elected officers are installed. The Presidents of the SAD during the past $25 \mathrm{yr}$ are presented in Table 14. Because the strength of our ADSA is so dependent on new members, the SAD deserves strong support by the ADSA membership.

\section{SIGNIFICANT CHANGES IN CONSTITUTION OF ADSA}

Ten of the most significant changes in our Constitution and Bylaws are indicated below.

1. The approval of membership in ADSA by the Board of Directors was removed.

2. The requirement for $5 \%$ of the voting membership being present at a meeting for amending the Constitution and Bylaws was removed. The requirement of $25 \%$ of the membership voting by mail ballot on Constitution and Bylaws also was removed.

3. A Postdoctoral Member class was developed for a person who holds a postdoctoral position and who is certified as such by a Professional Member. This Member is nonvoting, receives electronic access to the Journal of Dairy Science, and pays dues equal to $50 \%$ of that for the Professional Member.

4. The word chairman was changed throughout to chairperson.

5. The requirement to publish biographical sketches of nominees for ADSA Board of Directors in the Journal of Dairy Science was eliminated. Biographies of candidates now are submitted with ballots for election.

6. The opportunity to report official ADSA business via the Association's Internet Web site was approved. 
Table 10. Directors of the American Dairy Science Association, 1981-2005

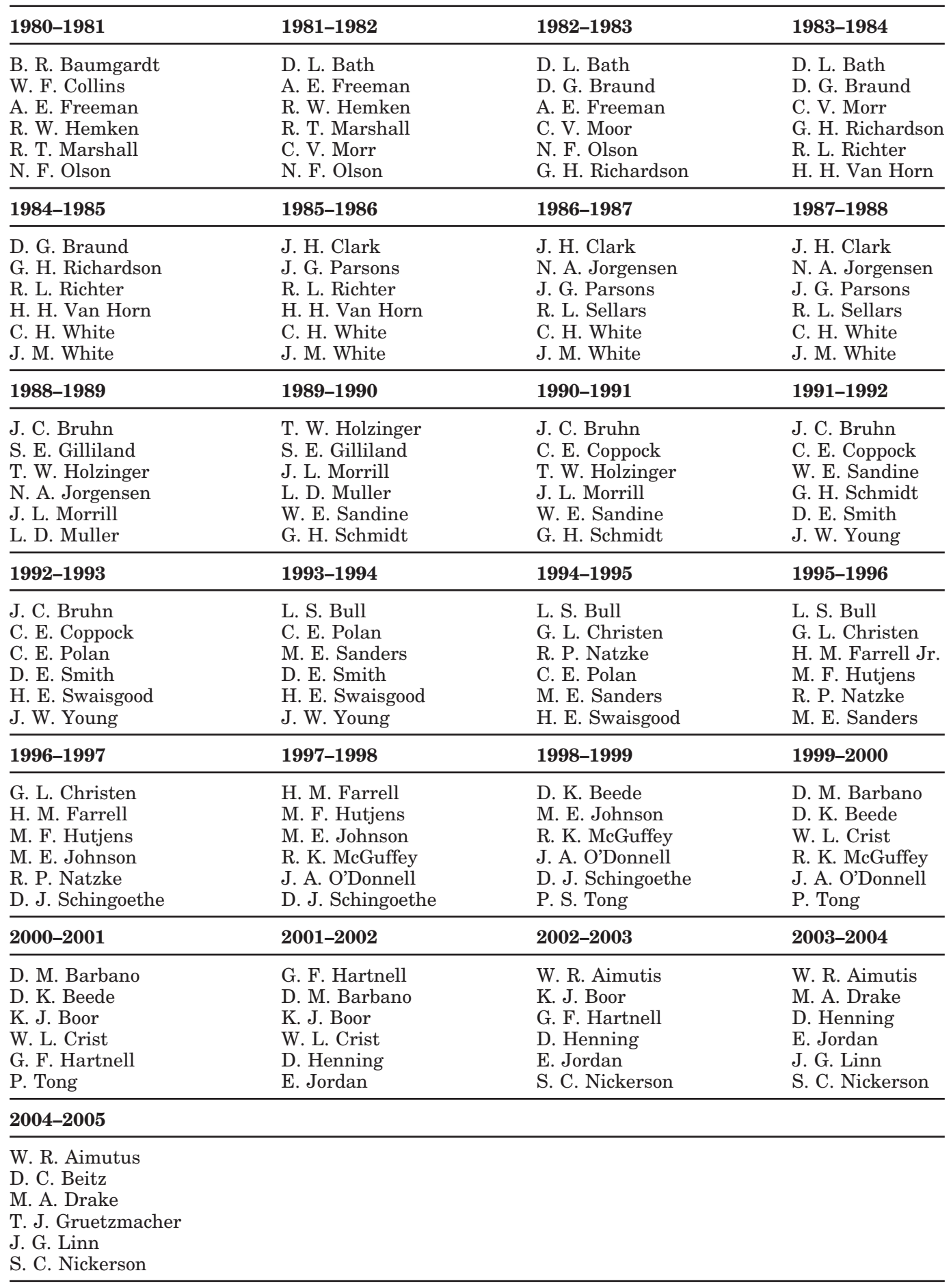

7. The Board of Directors shall establish annually an Internal Affairs Committee (vice president, 2 senior directors, and immediate past director from the division other than that of vice president) to consider business related to policies, affairs, and rewards.
8. Executive Secretary title was changed to Executive Director.

9. Vice President is to be chair of the Internal Affairs Committee.

10. Article VI was added to provide guidelines for the newly established ADSA Foundation. 
The above changes were noted by comparing the constitution/bylaws published in 1982 ADSA Directory and in 2005 on the ADSA website.

\section{ADSA JOINS FASS}

In the early 1980s, the Executive Committee of the board of directors and then the full board initiated discussion about forming a federation of the ADSA with ASAS, American Meat Science Association (AMSA), and the PSA. An Intersociety Council that consisted of 2 officers each from the ADSA, AMSA, ASAS, and PSA was formed but had no budget. Besides the publication of Food Animal Research Priorities, an important accomplishment of this Council was being a platform for greater intersociety interaction. Then, in 1988, the presidents or their representatives (C. Brent Theurer of ASAS, Jack Van Horn of ADSA, Michael Dikeman of AMSA, and David Snetsinger and Helene Cecil of PSA) developed an intersociety proposal that was approved by the boards of each of the 4 Intersociety Council members. The name of Federation of American Societies of Food Animal Sciences (FASFAS) was established in 1989. The 4 societies paid annual dues based on membership and named representatives to the FASFAS Board of Directors. Beginning in 1995, a committee explored development of a new structure and this discussion led to the formation of the Federation of Animal Science Societies (FASS) in 1998 and the dissolution of FASFAS. The Federation of Animal Science Societies would have a common management structure, but each individual member society would maintain its constitution, officers, budget, journal, foundation, awards program, and committee structure. The fact that the ADSA owned a building, provided management services for journals published by ASAS and PSA, and had a "healthy bank account" facilitated the establishment of FASS. The Federation purchased the building and assets originally owned by the ADSA at Savoy, IL, for its first headquarters. At this time, ADSA, ASAS, and PSA are founding member societies and AMSA is a client society. The presidents of FASFAS and FASS during their existence are named in Table 15. Thank you to these scientists for their committed leadership in this cooperative activity.

The benefits cited most often for forming FASS are to (1) provide a forum for discussion of common issues and for coordination of activities to benefit animal agriculture, (2) finance a Congressional Science Fellowship Program, (3) develop research priorities (follow-up of FAIR 95 and FAIR 2002), (4) increase efficiencies in use of resources of societies, (5) offer a stronger voice for animal agriculture in Washington, DC, and (6) finance an executive vice president in Savoy, IL, and a scientific liaison in Washington, DC. Robert Zimbelman was the first Scientific Liaison; Barbara Glenn followed him. Charles Sapp, formerly executive director of ADSA, was the first executive vice president for administration of FASS. Publication of the journals of ADSA, ASAS, PSA, and of several client organizations is managed by FASS. Animal scientists are now organized to cooperate more effectively for the betterment of animal agriculture globally.

\section{CONGRESSIONAL FELLOW PROGRAM}

To expand its activities in the public policy arena in support of animal agriculture, ADSA voted to join with ASAS, PSA, and the AMSA through FASFAS and later through FASS to provide financial support for a Congressional Science Fellowship Program. The concept was that the individual, often a new $\mathrm{PhD}$ degree graduate, would work with Congress on key issues of animal agriculture. Those who have served as Congressional Fellows are the following:

Angela Siemens, 1990-1991, University of Missouri Patrick Donnelly, 1991-1992, West Virginia University

Michael Westendorf, 1992-1993, University of Kentucky

Gary Ziehe, 1993-1994, Oklahoma State University

John Goldberg, 1994-1995, University of Vermont

Natalie Di Nicola, 1995-1996, University of Wisconsin

Ellen Bergfeld, 1996-1997, University of Nebraska

Caleb Gilchrist, 1997-1998, Texas A\&M University

Lisa Richards, 1998-1999, New Mexico State University

Jamie Jonker, 1999-2000, University of Maryland

Dana Hanson, 2000-2001, University of Nebraska

Dana Allen, 2002, University of Minnesota

John Dobrinsky, 2002, USDA-ARS

Brian Browker, 2003, Purdue University

Marcia S. Noble, 2004-2005, University of Illinois

Because of the success of the previous fellows, FASS plans to continue its support of this worthwhile activity.

\section{COLLABORATION WITH OTHER SOCIETIES}

The ADSA has continued to affiliate with a number of organizations besides those of FASS to expand its total effort to serve the membership and animal agriculture in general. To illustrate this effort, Table 16 lists the organizations to which ADSA provided representatives and/or paid dues to the following organizations in 1981 and in 2005. 


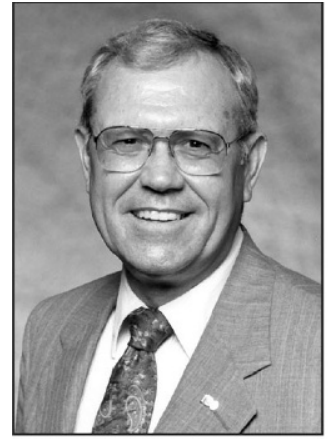

J. R. Campbell 65th President 1981
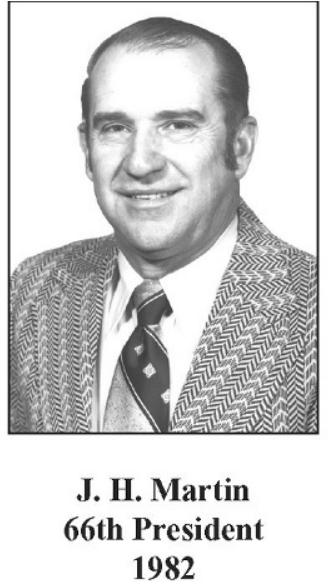

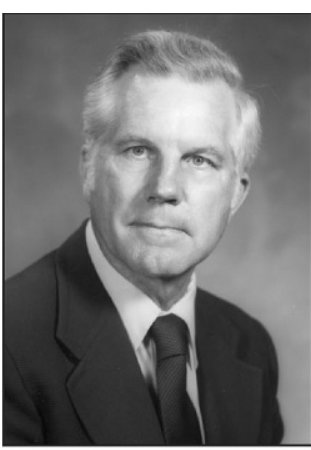

L. H. Schultz 67th President 1983

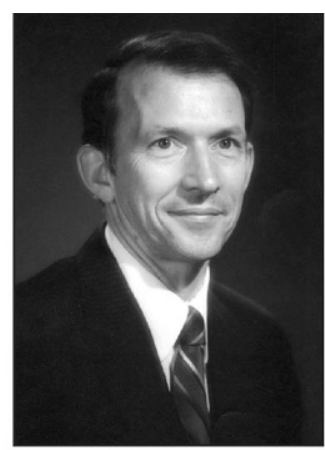

R. T. Marshall 68th President 1984

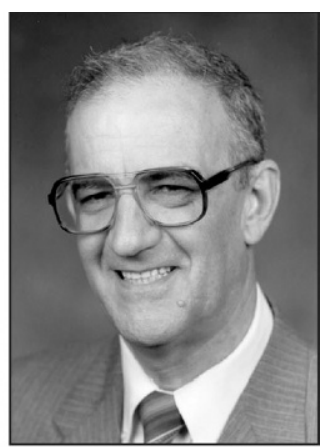

B. R. Baumgardt 69th President 1985

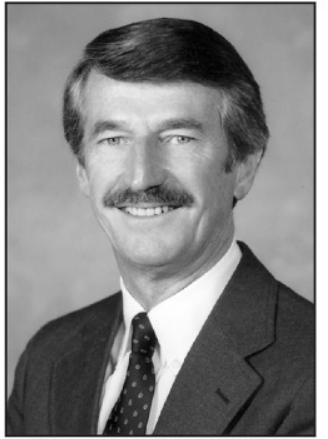

N. F. Olson

70th President 1986

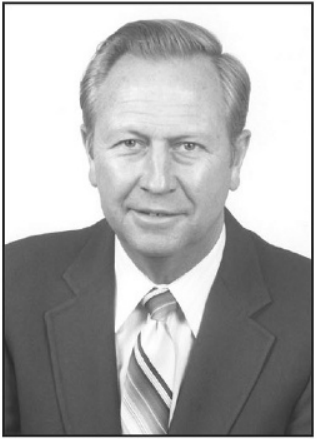

D. L. Bath 71st President 1987

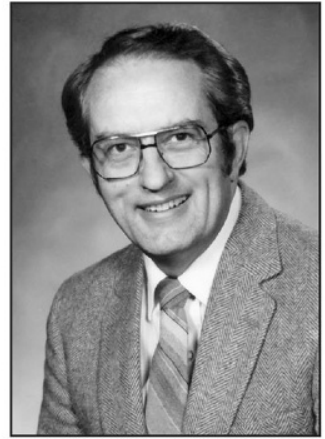

G. H. Richardson 72nd President 1988

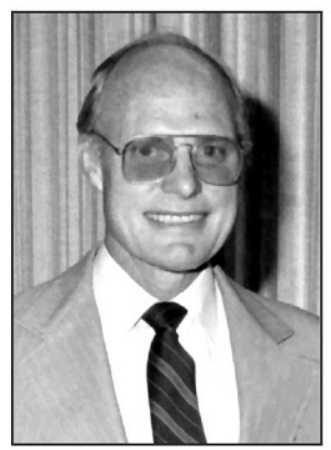

H. H. Van Horn 73rd President 1989

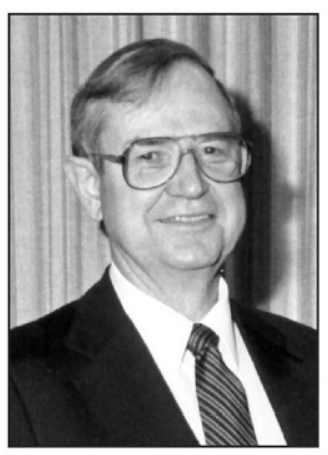
R. L. Sellars 74th President 1990

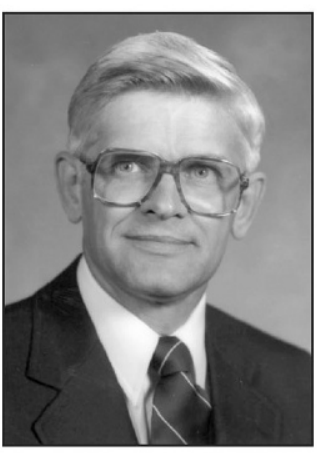

N. A. Jorgensen 75th President 1991

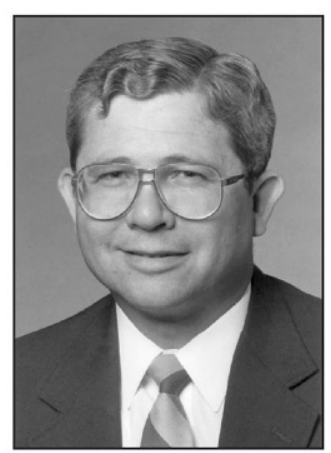

S. E. Gilliland 76th President 1992

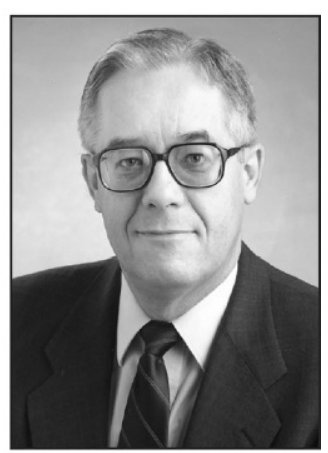

J. H. Clark 77th President 1993

Figure 1. American Dairy Science Association presidents from 1981 to 2006. 


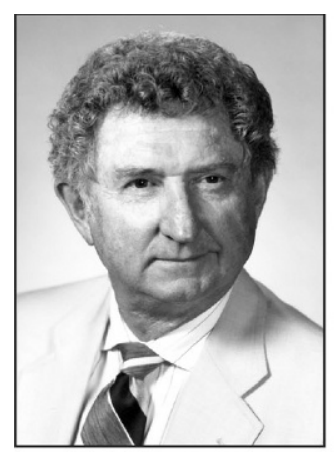

W. E. Sandine 78th President 1994

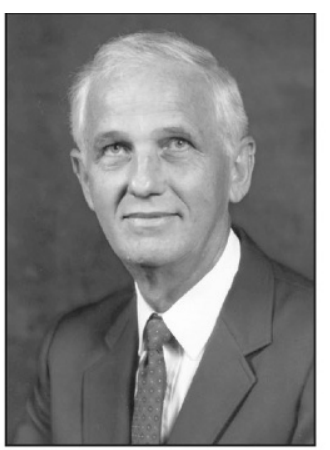

R. W. Hemken 79th President 1995

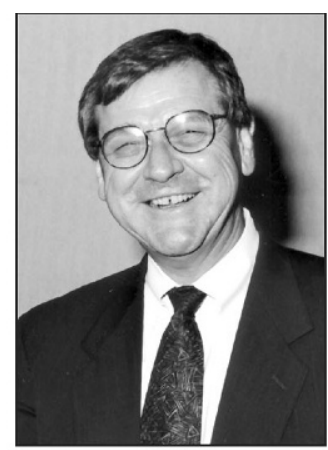

R. L. Richter 80th President 1996

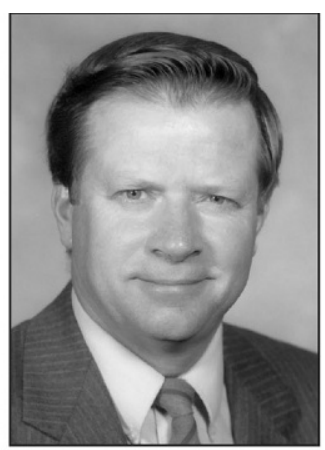

L. D. Satter 81st President 1997

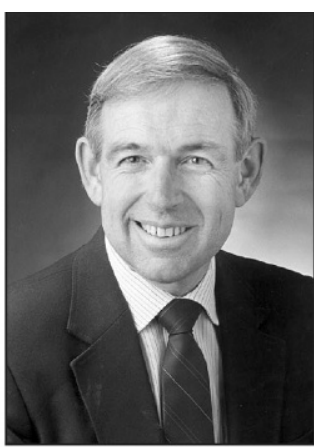

C. H. White 82nd President 1998

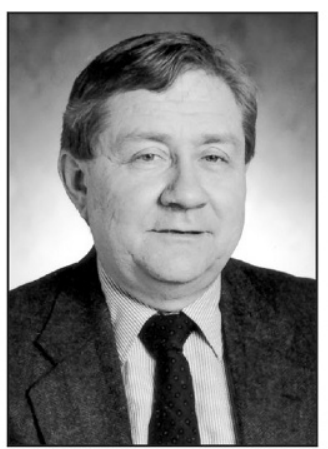

L. D. Muller 83rd President 1999

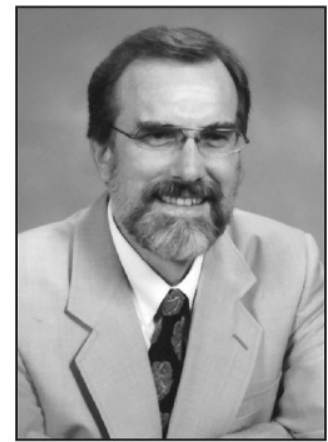

H. E. Swaisgood 84th President 2000

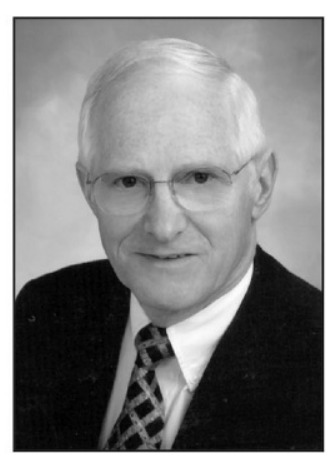

D. J. Schingoethe 85th President 2001

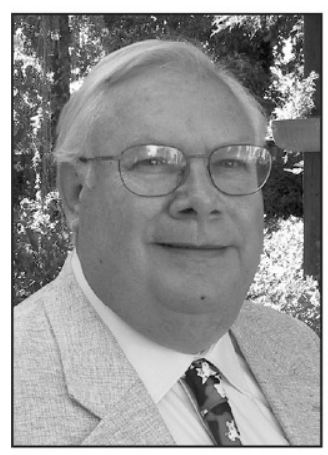

J. C. Bruhn 86th President 2002

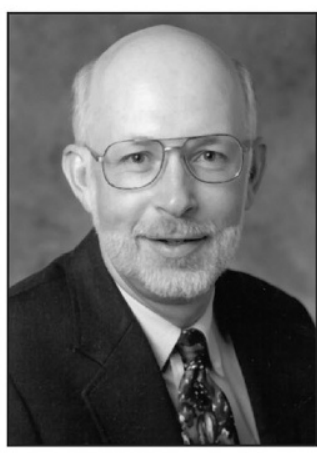

D. K. Beede 87th President 2003

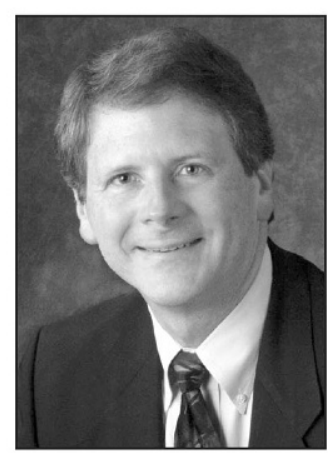
A. O'Donnell 88th President 2004

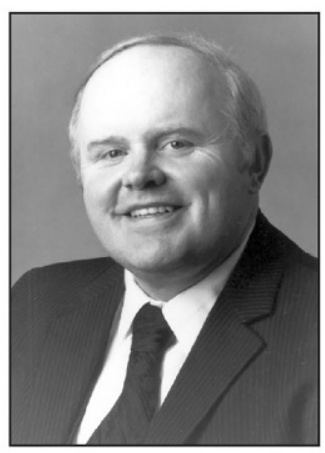

M. F. Hutjens 89th President 2005

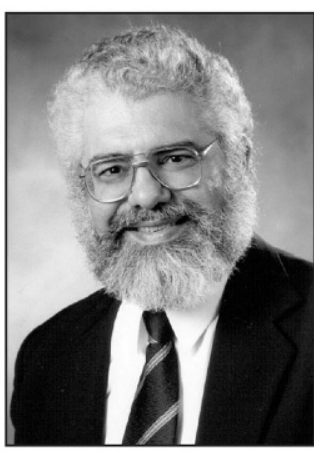

D. M. Barbano 90th President 2006

Figure 1 (Continued). American Dairy Science Association presidents from 1981 to 2006. 
Table 11. Distribution of the American Dairy Science Association presidents during its first century

\begin{tabular}{|c|c|c|c|c|c|}
\hline \multirow[b]{2}{*}{ State } & \multicolumn{4}{|c|}{25 -yr period } & \multirow[b]{2}{*}{ Total number } \\
\hline & $1906-1931^{1}$ & $1932-1956$ & $1957-1981$ & $1982-2005$ & \\
\hline & \multicolumn{4}{|c|}{$\longrightarrow$ (Number of presidents) } & \\
\hline New York & 3 & 3 & 3 & 0 & 9 \\
\hline Wisconsin & 0 & 2 & 2 & 5 & 9 \\
\hline Michigan & 2 & 3 & 2 & 1 & 8 \\
\hline Illinois & 0 & 2 & $2^{1}$ & 3 & 7 \\
\hline California & 0 & 1 & 2 & 3 & 6 \\
\hline District of Columbia & 0 & 2 & 2 & 0 & 4 \\
\hline Indiana & 1 & 1 & 1 & 1 & 4 \\
\hline North Carolina & 0 & 0 & 3 & 1 & 4 \\
\hline Pennsylvania & 2 & 0 & 1 & 1 & 4 \\
\hline Iowa & 1 & 0 & 2 & 0 & 3 \\
\hline Minnesota & 1 & 1 & 1 & 0 & 3 \\
\hline Missouri & 1 & 1 & 0 & 1 & 3 \\
\hline Ohio & 0 & 3 & 0 & 0 & 3 \\
\hline Connecticut & 1 & 0 & $1^{1}$ & 0 & 2 \\
\hline Kansas & 1 & 1 & 0 & 0 & 2 \\
\hline Florida & 0 & 1 & 0 & 1 & 2 \\
\hline Nebraska & 1 & 1 & 0 & 0 & 2 \\
\hline Texas & 0 & 0 & 1 & 1 & 2 \\
\hline Arizona & 0 & 0 & 1 & 0 & 1 \\
\hline Georgia & 0 & 1 & 0 & 0 & 1 \\
\hline Kentucky & 0 & 0 & 0 & 1 & 1 \\
\hline Mississippi & 0 & 0 & 0 & 1 & 1 \\
\hline Montana & 0 & 1 & 0 & 0 & 1 \\
\hline Oklahoma & 0 & 0 & 0 & 1 & 1 \\
\hline Oregon & 0 & 0 & 0 & 1 & 1 \\
\hline South Carolina & 0 & 0 & 0 & 1 & 1 \\
\hline South Dakota & 0 & 0 & 0 & 1 & 1 \\
\hline Utah & 0 & 0 & 0 & 1 & 1 \\
\hline Vermont & 1 & 0 & 0 & 0 & 1 \\
\hline Virginia & 0 & 0 & 1 & 0 & 1 \\
\hline Washington & 0 & 1 & 0 & 0 & 1 \\
\hline
\end{tabular}

\footnotetext{
${ }^{1}$ During this period, these presidents were in office more than one year. R. A. Pearson (2), C. H. Eckles (4), Hunziker (2), J. H. Frandsen (2), F. Rasmussen (2), W. A. Stocking (3), A. A. Borland (2), O. E. Reed (2), and G. C. White (2).
}

\section{THE ADSA FOUNDATION}

Under the leadership of R. Sellars as vice president and chair of the Internal Affairs Committee, the ADSA Foundation was established in 1990 by a vote of the ADSA membership. An initial financial contribution from Borden Co. jump-started its development. R. T. Marshall and his committee drafted the set of bylaws that were adopted. The Foundation is to receive private gifts for investment and program use toward its mission of providing educational and scientific activities for the betterment of the dairy industry and for the assurance of a safe, viable food supply to nourish the world. A 7member Board of Trustees oversees the activities of the Foundation and ensures that the Foundation remains steadfast to its mission. To accomplish its mission, the Foundation acquires and disseminates scientific knowledge and accurate information and enhances educational programs of the ADSA and the dairy industry as a whole.

Activities of the ADSA Foundation have expanded as the resources of the Foundation have grown since its establishment in 1990. Currently, the Foundation underwrites part of costs for the DISCOVER Conference Series and sponsors scientific symposia, Foundation Scholar Award, the Genevieve Christen Distinguished Undergraduate Student Award, and the auction and raffle as fundraisers. The DISCOVER Conferences are described in another section of this article. The ADSA Foundation Lectureships that enhanced our ADSA annual meetings for $5 \mathrm{yr}$ are listed in Table 17. In the early years, the Foundation also supported the lecture at the opening session of the annual ADSA meetings. With the holding of joint meetings with ASAS and other societies, this lecture is now supported otherwise. The ADSA Foundation established the Foundation Scholar Award in 1996 to recognize young scholars (within 10 yr after receiving the $\mathrm{PhD}$ degree) and their potential in research and educational leadership to promote the focus of attention on critical issues affecting the future of the dairy industry. One Scholar Award is to be given each year from the Dairy Foods and from the Production Divisions of ADSA. The previous Scholars are summa- 
Table 12. American Dairy Science Association division chairs, 19812005

\begin{tabular}{lll}
\hline Year & Dairy Foods & Production \\
\hline $1981-1982$ & R. L. Richter & D. G. Braund \\
$1982-1983$ & W. W. Coleman II & H. H. Van Horn \\
$1983-1984$ & C. H. White & G. T. Lane \\
$1984-1985$ & R. L. Sellars & J. H. Clark \\
$1985-1986$ & H. E. Swaisgood & N. A. Jorgensen \\
$1986-1987$ & J. C. Bruhn & L. D. Muller \\
$1987-1988$ & P. C. Vasavada & J. M. Elliot \\
$1988-1989$ & M. E. Sanders & J. W. Young \\
$1989-1990$ & G. L. Christen & L. D. Satter \\
$1990-1991$ & D. L. Willrett & C. E. Coppock \\
$1991-1992$ & J. F. Frank & C. E. Polan \\
$1992-1993$ & S. E. Barnard & R. A. Erdman \\
$1993-1994$ & R. J. Brown & M. F. Hutjens \\
$1994-1995$ & M. E. Johnson & D. E. Otterby \\
$1995-1996$ & J. V. McGregor & A. F. Kertz \\
$1996-1997$ & R. L. Selman & D. K. Beede \\
$1997-1998$ & K. A. Schmidt & W. L. Crist \\
$1998-1999$ & J. J. Warthesen & E. R. Jordan \\
$1999-2000$ & D. R. Henning & J. G. Linn \\
$2000-2001$ & T. J. Gruetzmacher & R. Shaver \\
$2001-2002$ & R. Jimenez-Flores & R. A. Cady \\
$2002-2003$ & M. Drake & C. G. Schwab \\
$2003-2004$ & S. A. Rankin & J. K. Drackley \\
$2004-2005$ & J. E. Schlesser & R. J. Grant \\
\hline
\end{tabular}

rized in Table 18. The Genevieve Christen Undergraduate Award was established in 1999 to recognize distinguished undergraduate students who have demonstrated leadership ability, academic achievement, involvement in the industry, and participation in the $\mathrm{SAD}$ and local club activities. Students who have re-
Table 14. Student affiliate division presidents, 1981-2005

\begin{tabular}{lll}
\hline Years & President & University \\
\hline $1980-1981$ & C. Rutter & Virginia Tech \\
$1981-1982$ & L. Bower & Penn State Univ. \\
$1982-1983$ & D. Alaniz & Louisiana State Univ. \\
$1983-1984$ & M. McGuire & Univ. of Illinois \\
$1984-1985$ & B. Moore & Univ. of Tennessee \\
$1985-1986$ & K. Kriebel & Penn State Univ. \\
$1986-1987$ & C. Hagenow & Univ. of Wisconsin-Madison \\
$1987-1988$ & T. Larson & Univ. of Minnesota \\
$1988-1989$ & P. Mitteness & Univ. of Minnesota \\
$1989-1990$ & N. Powel & Virginia Tech \\
$1990-1991$ & M. Haldiman & Univ. of Wisconsin-Madison \\
$1991-1992$ & T. Riggs & Ohio State Univ. \\
$1992-1993$ & D. Hovey & Iowa State Univ. \\
$1993-1994$ & S. Ellinghuysen & Univ. of Minnesota \\
$1994-1995$ & M. Ruby & Cal Poly State-San Luis Obispo \\
$1995-1996$ & M. Ramacker & Univ. of Missouri \\
$1996-1997$ & C. Bryant & Penn State Univ. \\
$1997-1998$ & R. Tanis & Penn State Univ. \\
$1998-1999$ & M. Laubach & North Dakota State Univ. \\
$1999-2000$ & M. Telfer & Univ. of Wisconsin-River Falls \\
$2000-2001$ & I. Norris & Louisiana State Univ. \\
$2001-2002$ & I. Peeler & Virginia Tech \\
$2002-2003$ & R. Blades & Louisiana State Univ. \\
$2003-2004$ & J. Moyer & Virginia Tech \\
$2004-2005$ & A. Lenkaitis & Univ. of Illinois \\
\hline & &
\end{tabular}

ceived this award are indicated in Table 19. Beginning in 2001, a traveling trophy (the Bob Sellar's cow carving) is presented annually to the student affiliate chapter that wins the SAD Quiz Bowl. Today, the Foundation sponsors the auction (started in 1993) and its associated raffle (started in 2003) as fund-raising activities

Table 13. American Dairy Science Association branch presidents, 1981-2005

\begin{tabular}{llll}
\hline Years & Northeast President & Southern President & Midwest President \\
\hline $1981-1982$ & M. W. Colburn & L. J. Bush & \\
$1982-1983$ & P. W. Moe & L. D. Guthrie & \\
$1983-1984$ & L. D. Muller & R. L. Richter & \\
$1984-1985$ & R. D. Smith & A. H. Rakes & \\
$1985-1986$ & H. F. Tyrrell & G. T. Lane & \\
$1986-1987$ & C. G. Schwab & J. A. Collins & \\
$1987-1988$ & S. J. Lyford & H. H. Van Horn & \\
$1988-1989$ & J. E. Nocek & D. W. Webb & \\
$1989-1990$ & G. W. Anderson & C. H. White & \\
$1990-1991$ & A. Pell & C. E. Polan & J. F. Keown \\
$1991-1992$ & M. A. Varner & F. Pardue & J. F. Keown \\
$1992-1993$ & L. E. Chase & J. R. Bishop & J. G. Linn \\
$1993-1994$ & J. E. Wohlt & B. W. Kellogg & D. J. Schingoethe \\
$1994-1995$ & K. R. Cummings & R. W. Hemken & J. L. Morrill \\
$1995-1996$ & L. Kung & L. W. Whitlow & M. F. Hutjens \\
$1996-1997$ & M. R. Stokes & G. L. Christen & M. A. Faust \\
$1997-1998$ & R. S. Kensinger & R. E. James & J. K. Drackley \\
$1998-1999$ & M. L. Westendorf & W. L. Crist & R. D. Shaver \\
$1999-2000$ & R. L. Baldwin & R. Gough & R. J. Grant \\
$2000-2001$ & S. M. Andrew & J. A. Bertrand & M. L. Eastridge \\
$2001-2002$ & P. S. Erickson & L. O. Ely & J. D. Quigley \\
$2002-2003$ & J. W. Comerford & D. D. Johnson & S. T. Franklin \\
$2003-2004$ & W. S. Cohick & J. K. Bernard & D. K. Combs \\
$2004-2005$ & R. A. Barczewski & B. A. Hopkins & L. Timms \\
$2005-2006$ & A. V. Capuco & D. Amaral-Phillips & P. Hoffman \\
\hline
\end{tabular}


Table 15. Presidents of Federation of American Societies of Food Animal Sciences (FASFAS) and Federation of Animal Science Societies $\left(\right.$ FASS) ${ }^{1}$

\begin{tabular}{|c|c|c|c|}
\hline 1989 & J. Van Horn ${ }^{2}$ & 1998 & L. D. Satter 3 \\
\hline 1990 & C. B. Theurer & 1999 & M. A. Ottinger \\
\hline 1991 & H. Cecil & 2000 & R. Natzke \\
\hline 1992 & M. Dikeman & 2001 & R. F. Wideman \\
\hline 1993 & S. Gilliland & 2002 & T. J. Klopfenstein \\
\hline 1994 & S. Curtis & 2003 & D. H. Beermann \\
\hline 1995 & B. Wentworth & 2004 & D. J. Schingoethe \\
\hline 1996 & R. Cassens & 2005 & J. W. Lauderdale \\
\hline 1997 & R. Hemken & & \\
\hline
\end{tabular}

${ }^{1}$ C. Sapp, former interim executive director for ADSA, was named as its executive director in 1998. In 2005, J. F. Baker, executive director of ASAS, was named CEO of FASS.

${ }^{2}$ First president of FASFAS.

${ }^{3}$ First president of FASS.

for the Foundation. The officers and the members of the Board of Trustees of the ADSA Foundation throughout its short history are presented in Table 20. Thank you to all the leaders of the Foundation for the significant enhancement of the ADSA and its annual meeting.

\section{DISCOVER CONFERENCES}

B. R. Baumgardt led the development of informal conferences called DISCOVER Conferences on Food Animal Agriculture that were patterned after the Gordon Conferences under the auspices of ADSA. The conferences, as proposed to the Board, would be a framework for critical thinking and discussion of innovative science rather than just reports on research findings. These guidelines were formulated. Conferences are to be held for 3 to $5 \mathrm{~d}$ in a retreat area. Attendance will be limited, and intellectual leaders will be invited for specific topics. Format will be a morning and evening session with an afternoon open for discussions and leisure activities. Participants will pay a registration fee, and outside support will be sought from granting agencies and companies. The ADSA Foundation will be the sponsor. The first DISCOVER Conference was held from June 1-4, 1998, at the Abe Martin Lodge in Brown County State Park (Nashville, IN) "to provide unique opportunities for interactions among scientists, industry, and the public that will synergize the development and use of science for the benefit of food animal industries and society." Topics for this and subsequent DISCOVER Conferences are presented in Table 21. Current interest in the DISCOVER Conferences on Food Animal Agriculture indicates that this program has a bright future.

\section{COMMUNICATION WITH THE MEMBERSHIP}

With the development of computers, new opportunities for communication with the membership became possible. The ADSA electronic homepage was developed during the early 1990 s to facilitate transfer of information from the headquarters and among the membership worldwide. Also, the publication of our Journal of Dairy Science online was accomplished in 1999. Payment of dues, registration for annual meetings, and voting now can be done electronically. So much information about the ADSA is now available at the nearest computer!

\section{THE JOURNAL OF DAIRY SCIENCE}

Today, the Journal of Dairy Science is the top-ranked journal of dairy science research in the world. Moreover,

Table 16. Organizations to which American Dairy Science Association has provided representatives and/ or paid dues in 1981 and 2005

\begin{tabular}{|c|c|c|}
\hline Organization & 1981 & 2005 \\
\hline American Association for Accreditation of Laboratory Animal Care ${ }^{1}$ & Yes & Yes \\
\hline American Association for the Advancement of Science ${ }^{1}$ & Yes & Yes \\
\hline American Feed Industry Association, Nutrition Council ${ }^{1}$ & Yes & Yes \\
\hline American Registry of Professional Animal Scientists & No & Yes \\
\hline American Society of Agronomy & Yes & No \\
\hline American Forage and Grassland Council & Yes & No \\
\hline Animal Agriculture Coalition & No & Yes \\
\hline Bovine Alliance on Management & No & Yes \\
\hline CoFARM & No & Yes \\
\hline Council for Agricultural Science and Technology ${ }^{1}$ & Yes & Yes \\
\hline Dairy Remembrance Fund & Yes & No \\
\hline International Dairy Federation ${ }^{1}$ & Yes & No \\
\hline National Association of Animal Breeders & Yes & No \\
\hline National Mastitis Council ${ }^{1}$ & Yes & Yes \\
\hline National Research Council Food and Nutrition Board & Yes & No \\
\hline Ralston Purina Fellowship Award & Yes & No \\
\hline 3-A Sanitary Standards Committee ${ }^{1}$ & Yes & Yes \\
\hline US Animal Health Association & Yes & No \\
\hline World Association for Animal Production ${ }^{1}$ & Yes & Yes \\
\hline
\end{tabular}

${ }^{1}$ Continous affiliation for 25 -yr period. 
Table 17. American Dairy Science Association Foundation lectureships at annual meetings of ADSA

\begin{tabular}{llll}
\hline $\begin{array}{l}\text { Lecture } \\
\text { No. }\end{array}$ & Year & Lecturer & Title \\
\hline 1 & 1993 & R. Heaney, Creighton Univ. & $\begin{array}{l}\text { Low calcium intake: Culprit in many chronic diseases. } \\
2\end{array}$ \\
3 & 1994 & O. Doering, Purdue Univ. & $\begin{array}{l}\text { Public perception and policy imperatives: Animal agriculture and the environment. } \\
\text { Serving the customer! Education's challenge for the future. }\end{array}$ \\
4 & 1995 & J. Gherty, Land O'Lakes, Inc. & $\begin{array}{l}\text { Agriculture's potential to help the nation meet sustainable environmental and food } \\
\text { production goals. }\end{array}$ \\
5 & 1997 & K. Matte, NDC of Canada & Repercussions down dairy lane. . The NAFTA/WTO factor. \\
\hline
\end{tabular}

it is one of the most highly ranked food technology journals. The Journal publishes results of original research in dairy foods, physiology and management; nutrition, feeding and calves; and genetics and breeding. As president of the ADSA, Frandsen made a plea in his presidential address to the Official Dairy Instructor's Association in 1913 for establishment of the Journal of Dairy Science. His wish for ADSA became reality because issue number 1 (84 pages) of volume 1 of the Journal of Dairy Science was published in May 1917 with J. H. Frandsen as Editor-in-Chief and 21 other scientists serving as a Board of Directors. Interestingly, the title to the first paper in the Journal was an "Address at dedication of new dairy building at University of Nebraska" by R. A. Pearson, the first president of the ADSA. The other 4 papers in the first issue dealt with bacterial analyses of milk, relations to breed associations, testing milk and cream for butterfat, and statistics of milk and cream regulations of the Official Dairy Instructors' Association, which was the name for ADSA before 1917. The 6 issues in volume 1 (532 pages) appeared in May, July, September, and November of 1917 and January and March 1918. Volume 2 was issued in January, March, May, July, September, and November of 1919 and contained 524 pages. Volume 3, issued every other month, contained 565 pages. It was not until 1940 when monthly issues of the Journal were published.

In 1981, an average of 32 papers were received each month and an average of 25 per month were published,

Table 18. American Dairy Science Association Foundation Scholar awards

\begin{tabular}{lll}
\hline Year & $\begin{array}{l}\text { Dairy Foods } \\
\text { Division Scholar }\end{array}$ & $\begin{array}{l}\text { Production } \\
\text { Division Scholar }\end{array}$ \\
\hline 1997 & S. Duncan & \\
1998 & F. Malcata & J. Drackley \\
1999 & N. Shah & D. Meyer \\
2000 & K. J. Boor & M. C. Lucy \\
2001 & J. A. Lucey & L. A. Holden \\
2002 & M. Wiedmann & W. Powers \\
2003 & M. Drake & K. A. Weigel \\
2004 & S. Rankin & M. Van Amburgh \\
2005 & L. Metzger & K. Knowlton \\
\hline
\end{tabular}

amounting to 2802 pages. The average time from submission to publication was 372 days. In 2005 , an average of 76 papers were received each month and an average of 40 were published, amounting to 4,451 pages. The average time from submission to publication was $214 \mathrm{~d}$. Thus, during the past $25 \mathrm{yr}$, there has been a gradual increase in the number of articles and pages published and a gradual decrease in time required for publication after submission. The decrease in publication time has been the result of an effort by editors and reviewers to become more prompt in their tasks and a streamlining of the publication process brought about, in part, by computerization of the whole process. Online submission and review of manuscripts began in July 2001. Online publication is provided by HighWire Press (Stanford, CA). Opportunities to publish Hot Topics, Short Communications and Technical Notes, Invited Reviews, and Letters to the Editors were added to the Journal in 1999. Interpretive Summaries of less than 100 words were included at the beginning of 2003 .

During the previous 25-yr period, dues for regular members, which provided for operations of the ADSA and a subscription to the Journal of Dairy Science, were $\$ 20$ per year. In 2005 , dues were $\$ 160$ per year with the option of paying only $\$ 110$ per year if the member chose to receive only the electronic copy of the Journal. The option for electronic copy became possible in 1999 . Subscription rates for institutions such as libraries to receive the Journal went from $\$ 30$ per year to $\$ 450$ per year during the 25-yr period. Page charges for publication in the Journal went from $\$ 30$ per page to $\$ 85$ per page during the same period. The Journal assists significantly in the sustenance of the ADSA.

Table 19. Genevieve Christen Undergraduate Student award

\begin{tabular}{ll}
\hline Year & Awardee \\
\hline 1999 & C. S. Albrecht, University of Minnesota \\
2000 & M. M. Birchen, University of Illinois \\
2001 & I. Norris, Louisiana State University \\
2002 & A. Iager, Purdue University \\
2003 & D. L. Zoellner, South Dakota State University \\
2004 & S. Vaughn, Iowa State University \\
2005 & S. Olson, University of Minnesota \\
\hline
\end{tabular}


Table 20. Board of trustees and officers of ADSA Foundation

\begin{tabular}{|c|c|c|c|}
\hline 1990-1991 & 1991-1992 & $1992-1993$ & 1993-1994 \\
\hline $\begin{array}{l}\text { R. L. Sellars } \\
\text { D. L. Bath } \\
\text { B } \\
\text { B. R. Baumgardt } \\
\text { J. R. Campbell } \\
\text { W. L. Dunkley } \\
\text { R. T. Marshall } \\
\text { N. F. Olson }\end{array}$ & $\begin{array}{l}\text { R. L. Sellars }{ }^{1} \\
\text { D. L. Bath } \\
\text { B. R. Baumgardt } \\
\text { J. R. Campbell } \\
\text { R. T. Marshall } \\
\text { G. Muck } \\
\text { N. F. Olson } \\
\text { L. H. Schultz }\end{array}$ & $\begin{array}{l}\text { R. L. Sellars }{ }^{1} \\
\text { R. T. Marshall } \\
\text { B. R. Baumgardt } \\
\text { N. A. Jorgensen } \\
\text { G. A. Muck } \\
\text { N. F. Olson } \\
\text { H. H. Van Horn }\end{array}$ & $\begin{array}{l}\text { R. L. Sellars }{ }^{1} \\
\text { R. T. Marshall } \\
\text { B. R. Baumgardt } \\
\text { N. A. Jorgensen } \\
\text { G. A. Muck } \\
\text { N. F. Olson } \\
\text { H. H. Van Horn }\end{array}$ \\
\hline 1994-1995 & 1995-1996 & 1996-1997 & 1997-1998 \\
\hline $\begin{array}{l}\text { R. L. Sellars }{ }^{1} \\
\text { R. T. Marshall } \\
\text { D. L. Bath } \\
\text { B. R. Baumgardt } \\
\text { N. A. Jorgensen } \\
\text { G. A. Muck } \\
\text { N. F. Olson } \\
\text { H. H. Van Horn }\end{array}$ & $\begin{array}{l}\text { H. H. Van Horn }{ }^{1} \\
\text { R. L. Sellars } \\
\text { D. L. Bath } \\
\text { B. R. Baumgardt } \\
\text { N. A. Jorgensen } \\
\text { G. A. Muck }\end{array}$ & $\begin{array}{l}\text { H. H. Van Horn }{ }^{1} \\
\text { R. L. Sellars }{ }^{4} \\
\text { J. H. Clark } \\
\text { S. E. Gilliland } \\
\text { R. W. Hemken } \\
\text { N. A. Jorgensen } \\
\text { G. A. Muck } \\
\text { W. E. Sandine }\end{array}$ & $\begin{array}{l}\text { S. E. Gilliland }{ }^{1} \\
\text { R. L. Sellars }{ }^{4} \\
\text { D. G. Braund } \\
\text { J. H. Clark } \\
\text { R. W. Hemken } \\
\text { N. A. Jorgensen } \\
\text { G. A. Muck } \\
\text { W. E. Sandine } \\
\text { D. L. Willrett }\end{array}$ \\
\hline 1998-1999 & 1999-2000 & 2000-2001 & 2001-2002 \\
\hline $\begin{array}{l}\text { J. H. Clark }{ }^{1} \\
\text { R. W. Hemken }{ }^{2} \\
\text { W. E. Sandine } \\
\text { H. H. Van Horn } \\
\text { D. G. Braund } \\
\text { D. E. Gilliland } \\
\text { S. E. A. Muck } \\
\text { G. D. Satter }\end{array}$ & $\begin{array}{l}\text { R. M. Hemken }{ }^{1} \\
\text { G. A. Muck }{ }^{2} \\
\text { W. E. Sandine } \\
\text { H. H. Van Horn } \\
\text { D. G. Braund } \\
\text { J. H. Clark } \\
\text { S. E. Gilliland } \\
\text { L. D. Satter }\end{array}$ & $\begin{array}{l}\text { G. A. Muck, Chair } \\
\text { L. D. Satter } \\
\text { S. E. Gilliland } \\
\text { H. H. Van Horn } \\
\text { D. G. Braund } \\
\text { J. H. Clark } \\
\text { R. W. Hemken } \\
\text { W. E. Sandine }\end{array}$ & $\begin{array}{l}\text { L. D. Satter }{ }^{1} \\
\text { J. H. Clark } \\
\text { C. H. White } \\
\text { R. L. Richter } \\
\text { R. G. Braund } \\
\text { D. } \\
\text { R. W. Hemken } \\
\text { A. F. Kertz } \\
\text { G. A. Muck }\end{array}$ \\
\hline 2002-2003 & 2003-2004 & 2004-2005 & 2005-2006 \\
\hline $\begin{array}{l}\text { A. F. Kertz }{ }^{1} \\
\text { R. K. McGuffey } \\
\text { C. H. White } \\
\text { R. L. Richter } \\
\text { R. } \\
\text { D. Barbano } \\
\text { K. R. Cummings } \\
\text { T. M. Gilmore }\end{array}$ & $\begin{array}{l}\text { A. F. Kertz } \\
\text { R. K. McGuffey } \\
\text { C. H. White } \\
\text { C. } \\
\text { R. L. Richter } \\
\text { D. Barbano } \\
\text { L. Hansen } \\
\text { L. Metzger }\end{array}$ & $\begin{array}{l}\text { A. F. Kertz } \\
\text { R. K. McGuffey } \\
\text { Le } \\
\text { L. Metzger } \\
\text { E. R. Jordan } \\
\text { W. R. Aimutis } \\
\text { L. B. Hansen } \\
\text { D. R. Henning }\end{array}$ & $\begin{array}{l}\text { A. F. Kertz } \\
\text { L. B. Hansen } \\
\\
\text { L. Metzger } \\
\text { E. R. Jordan } \\
\text { W. R. Aimutis } \\
\text { D. R. Henning } \\
\text { A. Schultz }\end{array}$ \\
\hline
\end{tabular}

To commemorate the first $50 \mathrm{yr}$ of progress in teaching, research, and extension of dairy science, the Journal of Dairy Science published a Golden Jubilee Issue containing about 290 pages of text. The 75th Anniversary of ADSA was celebrated, in part, by publication of a Diamond Jubilee Issue of 656 pages of scientific information. This article is the leadoff paper in a similar issue to commemorate the centennial year of the ADSA.

The achievement of a highly ranked scientific journal for publication of dairy science information required the efforts of dedicated editors-in-chief of the Journal of Dairy Science. In 1996-1997, senior editors for newly formulated sections based on subject matter (dairy foods; physiology and management; nutrition, feeding, and calves; and genetics and breeding) were named to assist the editor-in-chief. The names of the editors-inchief of the Journal from 1981 to the present time are listed in Table 22. Thank you to this group of scientists who have been so dedicated to the excellence of the Journal of Dairy Science.

\section{INTERNATIONALIZATION OF THE ADSA}

The outstanding scientific reputation of our membership and of our Journal of Dairy Science has led to an increased number of memberships in the ADSA and of published papers in the Journal from non-US scientists. In 2003 , about $30 \%$ of ADSA members were from outside of the United States. The top 9 non-US countries that contributed to $30 \%$ of total membership of ADSA in 
Table 21. DISCOVER conferences on food animal agriculture

\begin{tabular}{|c|c|c|}
\hline $\begin{array}{l}\text { Conference } \\
\text { no. }^{1}\end{array}$ & Title & Date \\
\hline First & Research solutions to assure microbial safety of foods of animal origin & June 1-4, 1998 \\
\hline Second & Transition cows & August 29-September 1, 1999 \\
\hline Fourth & Probiotics for food animals & September $10-13,2000$ \\
\hline Fifth & $\begin{array}{l}\text { Milk, dairy ingredients, and dairy foods for the new decade: Developing } \\
\text { new composition, nutritional, and functional targets for tomorrow's consumer }\end{array}$ & January $20-23,2002$ \\
\hline Sixth & Nitrogen losses to the atmosphere from livestock and poultry operations & April 28-May 2, 2002 \\
\hline Ninth & $\begin{array}{l}\text { Protecting and managing animal genetic resources for future generations: } \\
\text { The next step }\end{array}$ & November $2-5,2004$ \\
\hline Tenth & $\begin{array}{l}\text { Improving health through dairy foods: New technologies, new research, } \\
\text { and new directions }\end{array}$ & April 10-13, 2005 \\
\hline Eleventh & Amino acid requirements of dairy cows & August $28-31,2005$ \\
\hline
\end{tabular}
was held at Stone Mountain, GA.

2003 (expressed as percentage of non-US membership) were as follows: Canada (19\%), Japan (12\%), Italy (6\%), France (5\%), Spain (5\%), Germany (5\%), Australia (4\%), S. Korea (4\%), and the Netherlands (4\%). Other European countries (16\%), Latin America (9\%), Asia (5\%), and other countries provided the remaining international members. About 50\% of the manuscripts published in the 2003 issues of the Journal of Dairy Science were from international scientists; for comparison, in $1994,30 \%$ of publications were from international scientists. In 2003, one-fifth of the published international manuscripts were derived from collaborative research by US and non-US scientists. Canada (18\%), United Kingdom (9\%), Spain (9\%), France (9\%), Denmark (7\%), and Netherlands $(6 \%)$ were the major contributors of international papers published in the Journal in 2003. Other European countries (24\%), Latin America (4\%), and all other countries (14\%) contributed the remainder of international papers. The recent increase in percentage of international members and the linear increase

Table 22. Journal of Dairy Science editors-in-chief

\begin{tabular}{llll}
\hline Year & Name & Year & Name \\
\hline 1981 & L. D. McGilliard & $1992-1993$ & J. B. Holter \\
1982 & L. D. McGilliard & $1993-1994$ & J. W. Fuquay \\
1983 & L. D. McGilliard & $1994-1995$ & D. B. Emmons \\
$1983-1984$ & L. D. McGilliard & $1995-1996$ & L. B. Hansen \\
$1984-1985$ & C. A. Ernstrom & $1996-1997$ & J. W. Fuquay \\
$1985-1986$ & C. A. Ernstrom & $1997-1998$ & J. W. Fuquay \\
$1986-1987$ & C. A. Ernstrom & $1998-1999$ & J. W. Fuquay \\
$1987-1988$ & C. A. Ernstrom & $1999-2000$ & J. W. Fuquay \\
$1988-1989$ & G. H. Schmidt & $2000-2001$ & J. W. Fuquay \\
$1989-1990$ & G. H. Schmidt & $2001-2002$ & J. W. Fuquay \\
$1990-1991$ & R. L. Richter & $2002-2003$ & S. C. Nickerson \\
$1991-1992$ & G. R. Wiggans & $2003-2004$ & S. C. Nickerson \\
& & $2004-2005$ & S. C. Nickerson \\
\hline
\end{tabular}

in percentage of international published papers since 1994 suggest increased participation by international scientists in our Association for the next several years as well. The International Reception has continued as an integral part of the annual ADSA meeting.

\section{A NEW EXPERIENCE-WITHSTANDING A CIVIL ACTION SUIT}

The ADSA was sued by 2 commercial companies in US District Court in Minneapolis, MN, on February 1, 1981 , seeking $\$ 10$ million in damages on each of 7 counts of alleged libel and disparagement. Also sued were the Regents of Texas A\&M University and the author of a research paper published in the September 1980 issue of the Journal of Dairy Science.

Holding firm to the validity of the peer-review process enabling the publishing of the findings of scholarly research and believing it imperative to preserve the academic freedom of the author, the Board of Directors voted unanimously to defend ADSA interests. Legal counsel was available to the reviewers, editors, and ADSA employees for all depositions taken in Orlando and Gainesville, FL as well as in College Station, TX, and Washington, DC. This suit caused a considerable drain on the ADSA budget.

At the June 1982 annual meeting, an announcement of an increase in dues of $\$ 5$ per year (from $\$ 20$ to $\$ 25$ ) was followed by a status report on the lawsuit filed in 1981. The motion to dismiss the suit was denied, and during the year the suit was increased to 9 counts, 8 of which were for $\$ 10$ million each and 1 count was for $\$ 7$ million, totaling $\$ 87$ million. Legal costs through June 1982 were $\$ 28,000$. In December 1982, the plaintiffs proposed an out-of-court settlement and, after con- 


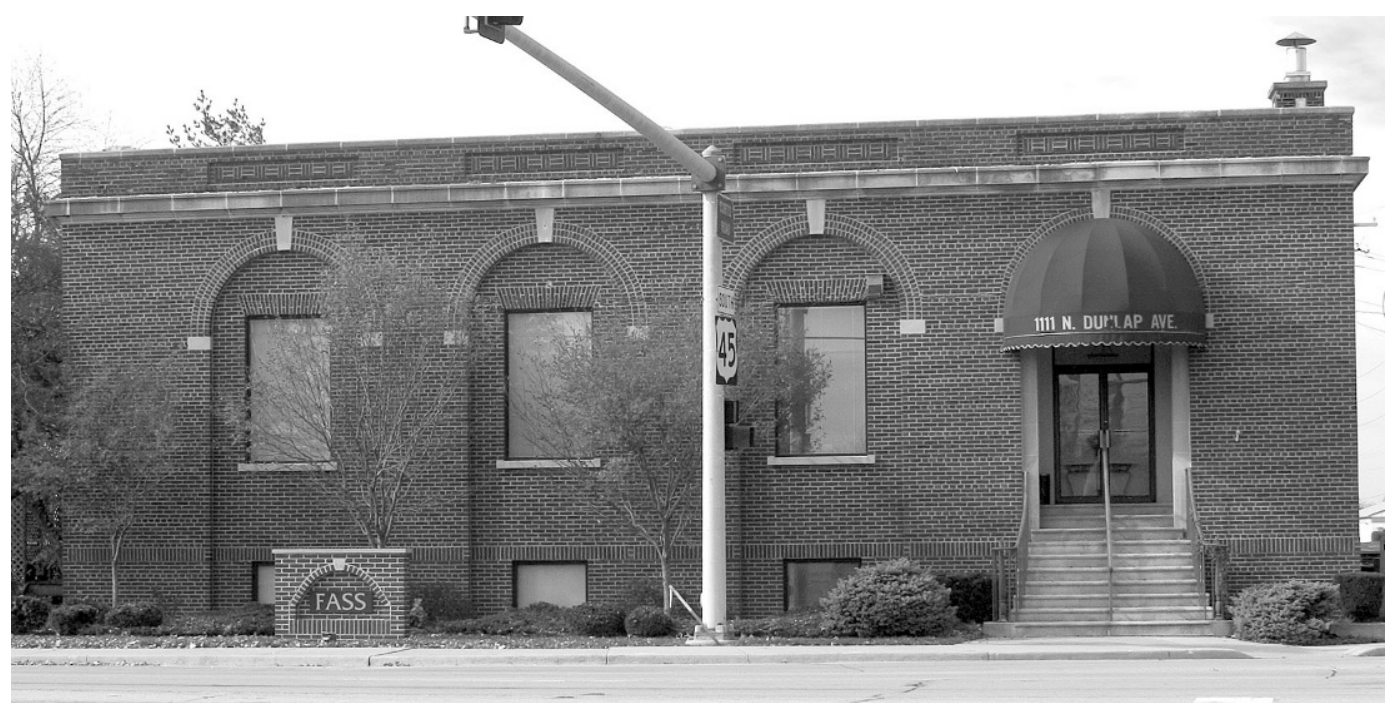

Figure 2. The American Dairy Science Association headquarters building in Savoy, IL.

siderable negotiations, the Board of Directors agreed unanimously on the terms of the settlement. At the June 1983 annual meeting, President L. H. "Bud" Schultz reported on the legal steps actively pursued to resolve the case.

The settlement stipulated no admission of guilt or wrongdoing by ADSA and a monetary settlement of $\$ 10,000$. President Schultz emphasized that settlement was made solely because of concern over the high cost and diversion of effort involved in pursuing the suit. It was felt that ADSA could win the suit, but it might have lasted for years and would be cost prohibitive. Even with the settlement, direct costs of the suit exceeded $\$ 70,000$. To afford some future protection, ADSA obtained an insurance policy to cover legal defense in a civil action suit.

The ADSA continues to maintain that it is the responsibility of dairy scientists to conduct research on all aspects of dairy science including commercial products and to evaluate and publish all worthy research in an unbiased manner. Despite this unfortunate and frustrating experience for ADSA, the suit was settled without compromising any principles regarding publication of worthy research.

\section{ADSA MOVES TO NEW SITE}

The ADSA began its fourth $25 \mathrm{yr}$ of existence in its own building at 309 West Clark Street in Champaign, IL. This is where the business of running the ADSA and the management services for the Journal of Dairy Science were conducted. In addition, ADSA provided management services for publication of the Journal of
Animal Science for ASAS, Poultry Science for PSA, and Journal of Reproduction for the Society for the Study of Reproduction, and for publications for the North Central Weed Control Conference, Southern Weed Science Society, and Weed Science Society of America. Later, services were provided for a few years for publication of the Journal of Nutrition. In 2006, FASS continues to provide management services for publications of the ASAS, PSA, and ADSA as well as for publication of the Professional Animal Scientist for the American Registry for Professional Animal Scientists.

After much discussion by the Board of Directors during the early $1990 \mathrm{~s}$, a decision was made to purchase a larger building at 1111 North Dunlap Avenue in Savoy, IL, which is located on the southern fringe of Champaign, and to sell the Clark Street building. The staff moved the ADSA office to the Savoy site in September 1995. New software and hardware was installed in the new building to maintain the membership database as well as history, annual meeting registration, publication purchases, and invoicing. By 1997, the directory of members was online and submission and review of manuscripts and publication of the Newsletter and ADSA Today was performed electronically through the ADSA website. The new headquarters building is shown in Figure 2.

\section{CENTENNIAL CELEBRATION}

The ADSA will celebrate its Centennial in 2006 at its annual meeting in Minneapolis, MN. This article on the history of ADSA from 1981 to 2005 is part of a special issue of the Journal of Dairy Science that up- 
Table 23. Deaths of Members of ADSA from 1980 to 2005

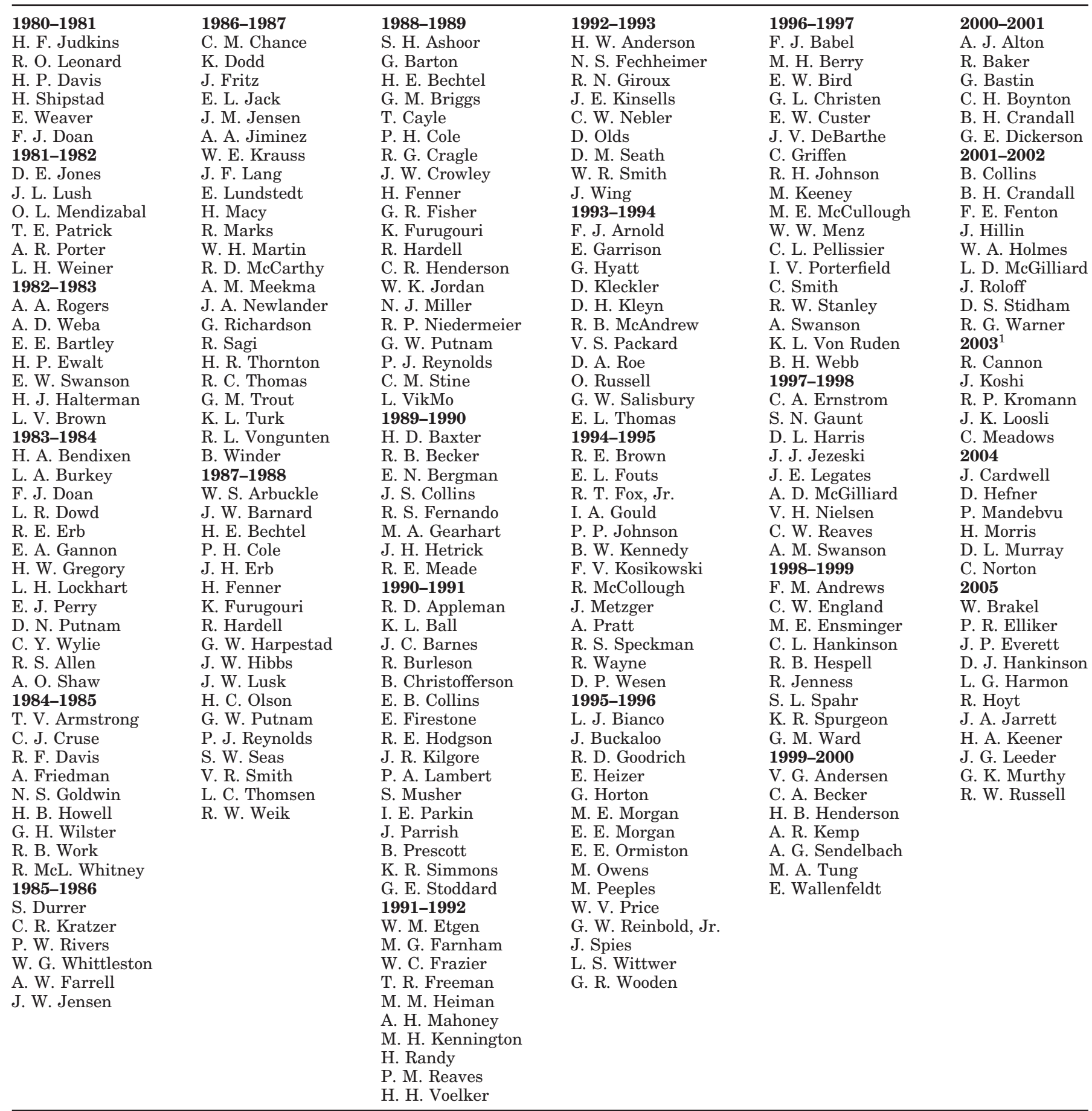

${ }^{1}$ Recording of deaths changed to the calendar basis beginning in 2003.

dates readers with scientific information in Dairy Production and Dairy Foods since the Diamond Jubilee edition in 1981. A second publication will be a historical book on advances in the production and use of milk as a food. This "coffee table" book also will provide a pictorial history and narrative of the contributions of the ADSA to the dairy industry during the past $100 \mathrm{yr}$. A centennial ADSA logo has been developed. A video that chronicles contributions of institutions and individuals during the first $100 \mathrm{yr}$ will also be available. Moreover, animal, dairy, and food science departments will be providing exhibits of their organization's contributions to ADSA 
and the dairy industry for the Centennial meeting of ADSA. Clearly, we have much to celebrate at the annual meeting in 2006 !

\section{IN REMEMBRANCE}

We should all take a moment of silence to remember with fondness those members of ADSA who have died during the 25-yr period from 1981 to 2005 (Table 23).

\section{SUMMARY}

Through the previous $25 \mathrm{yr}$ of its history, the ADSA continues to accomplish its mission of providing leadership in scientific and technical support to sustain and grow the global dairy industry through generation, dissemination, and exchange of information and services. The Association, along with ASAS and PSA, is a member of FASS, whose mission is to strengthen the common interests and collective good of member societies through a unified science-based voice that supports animal agriculture, animal products, and food systems globally via effective, efficient, and economical administrative and programmatic services. Highlights during the past $25 \mathrm{yr}$ include the transfer of journal publication and much of public policy activities to FASS, development of postdoctorate member status, greater internationalization of our membership and Journal, improved scientific stature of our Journal, expanded recognition of members, excellence in research, teaching, and outreach of our members, development of an ADSA Foundation for expanded support of ADSA activities, and a movement of the annual meetings from university campuses to convention centers. The future of the
ADSA is bright, and the history of the next 25 years of the ADSA promises to be exciting as well.

Be well
Do good work
Keep in touch

-Garrison Keillor

\section{ACKNOWLEDGMENTS}

The authors acknowledge the gracious assistance in gathering data for the manuscript by ADSA Executive Director Brenda Carlson. The excellence of Julie Roberts in typing the manuscript is greatly appreciated.

\section{REFERENCES}

American Dairy Science Association. 2002. Revised constitution of the American Dairy Science Association and Revised bylaws of the American Dairy Science Association. Online. www.adsa.org/ADSABylaws

Anonymous. 1981a. Special award of appreciation presented to G. Malcolm Trout, historian. J. Dairy Sci. 64:1895-1896.

Anonymous. 1981b. Special award of appreciation presented to Claude J. Cruse, executive secretary. J. Dairy Sci. 64:1897.

Anonymous. 1982. Revised constitution and bylaws of the American Dairy Science Association. ADSA Directory.

Anonymous. 1985. Memorial: Claude J. Cruse. J. Dairy Sci. 68:1320-1321.

Dunkley, W. L. 1989. Business management of American Dairy Science Association: A historical review. J. Dairy Sci. 72:288-304.

Musgrave, S. D. 1964. The ADSA bell. J. Dairy Sci. 77:1154.

Trout, G. M. 1956. Fifty years of the American Dairy Science Association. J. Dairy Sci. 39:625-650.

Trout, G. M. 1961. The awards of the American Dairy Science Association: A review. J. Dairy Sci. 44:955-964.

Trout, G. M. 1964. Development of the student affiliate program. J. Dairy Sci. 47:1439-1447.

Trout, G. M. 1981. The American Dairy Science Association: The third twenty-five years, 1957-1981. J. Dairy Sci. 64:876-899. 